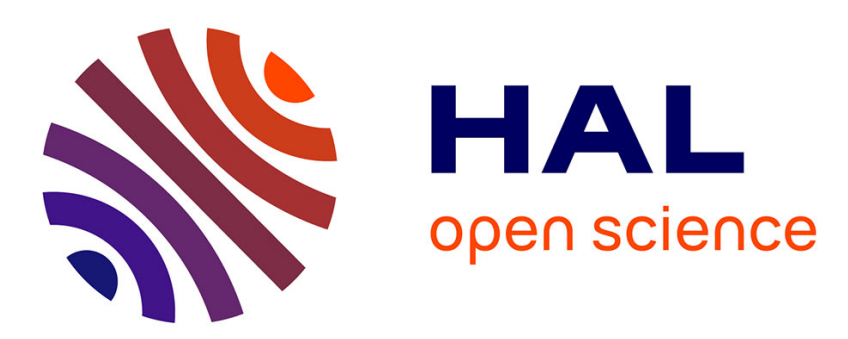

\title{
Passive simulation of the nonlinear port-Hamiltonian modeling of a Rhodes Piano
}

\author{
Antoine Falaize, Thomas Hélie
}

\section{To cite this version:}

Antoine Falaize, Thomas Hélie. Passive simulation of the nonlinear port-Hamiltonian modeling of a Rhodes Piano. 2016. hal-01390534

\section{HAL Id: hal-01390534 \\ https://hal.science/hal-01390534}

Preprint submitted on 2 Nov 2016

HAL is a multi-disciplinary open access archive for the deposit and dissemination of scientific research documents, whether they are published or not. The documents may come from teaching and research institutions in France or abroad, or from public or private research centers.
L'archive ouverte pluridisciplinaire HAL, est destinée au dépôt et à la diffusion de documents scientifiques de niveau recherche, publiés ou non, émanant des établissements d'enseignement et de recherche français ou étrangers, des laboratoires publics ou privés. 


\title{
Passive simulation of the nonlinear port-Hamiltonian modeling of a Rhodes Piano
}

\author{
Antoine Falaize ${ }^{1, *}$, Thomas Hélie ${ }^{1, *}$ \\ Project-Team S3 (Sound Signals and Systems) and Analysis/Synthesis team, Laboratory of \\ Sciences and Technologies of Music and Sound (UMR 9912), IRCAM-CNRS-UPMC, \\ 1 place Igor Stravinsky, F-75004 Paris
}

\begin{abstract}
This paper deals with the time-domain simulation of an electro-mechanical piano: the Fender Rhodes. A simplified description of this multi-physical system is considered. It is composed of a hammer (nonlinear mechanical component), a cantilever beam (linear damped vibrating component) and a pickup (nonlinear magneto-electronic transducer). The approach is to propose a power-balanced formulation of the complete system, from which a guaranteed-passive simulation is derived to generate physically-based realistic sound synthesis.

Theses issues are addressed in four steps. First, a class of Port-Hamiltonian Systems is introduced: these input-to-output systems fulfill a power balance that can be decomposed into conservative, dissipative and source parts. Second, physical models are proposed for each component and are recast in the port-Hamiltonian formulation. In particular, a finite-dimensional model of the cantilever beam is derived, based on a standard modal decomposition applied to the Euler-Bernoulli model. Third, these systems are interconnected, providing a nonlinear finite-dimensional Port-Hamiltonian System of the piano. Fourth, a passive-guaranteed numerical method is proposed.

This method is built to preserve the power balance in the discrete-time domain, and more precisely, its decomposition structured into conservative, dissipative and source parts. Finally, simulations are performed for a set of physical parameters, based on empirical but realistic values. They provide a variety of audio signals which are perceptively relevant and qualitatively similar to some signals measured on a real instrument.
\end{abstract}

Keywords: Passive Modeling, Numerical Methods, Port-Hamiltonian Systems, Multiphysics System, Time Domain Simulation.

\footnotetext{
${ }^{*}$ Corresponding author

Email address: antoine.falaize@gmail.com (Antoine Falaize)

${ }^{1}$ The contribution of both authors has been done at IRCAM, Paris, within the context of the French National Research Agency sponsored project HAMECMOPSYS. Further information is available at http://www.hamecmopsys.ens2m.fr. The $\mathrm{PhD}$ thesis of A. Falaize is funded by the EDITE school from Paris.
} 


\section{Introduction}

Sound synthesis based on physical modeling aims at recovering natural behaviors of existing (or imaginary) instruments. This includes transients, effects due to damping phenomena, timbre variations due to nonlinearities, etc. How5 ever, since the models are nonlinear, guaranteeing numerical stability is not straightforward. In this context, approaches based on energy have been developed and applied to simulate musical instruments [1, 2, 3, 4]. The principle relies on passivity: conservative (or dissipative) phenomena make the energy time-variation equal to (or lower than) the power received from external sources. Passivity provides energy bounds, from which state bounds stem, so that preserving this property in simulations can be used to address stability issues.

Most of these methods involve space-time discretizations and numerical schemes that are devoted to handle conservative problems and that can successfully be applied to more realistic dissipative models. Technically, these methods

15 are usually designed in such a way that a numerical power balance is fulfilled for the discretized quantities (e.g. as the product of discrete velocities and forces). In this paper, this point of view is modified and handled in two steps: (I) derive a passive model in the continuous time-domain, through conservative interconnections of passive elementary components; (II) transpose the complete

20 system in discrete-time domain in such a way that the original power balance is naturally fulfilled and passivity is naturally preserved.

Numerous methods are available to reach step (I). They can be divided in two main classes: (WS) wave scattering methods and (KV) Kirchhoff's variables 25 methods (see e.g. [5]). Mixed WS/KV methods have also been proposed [6]. Several methods are also available for step (II). Wave-digital filters (WDF) [7] and digital wave-guide (DWG) $[\underline{8}$ are commonly used in audio and acoustic applications. These formalisms belong to the class of WS methods. They allow block-based modeling approaches [6], by introducing links that mimic the serial so and parallel connections, and result in passive models for linear systems [9]. WDF and DWG approaches lead to realizable and explicit numerical systems. They are appreciated in real-time sound synthesis applications. However, their benefits are lost for nonlinear systems.

35 This paper deals with a nonlinear system: the Fender Rhodes piano. Its passive modeling is derived in the class of Port-Hamiltonian Systems, introduced in the 1990s [10, 11, 12. These systems can be considered as an extension of Hamiltonian systems [13] in the sense that these dynamical systems can be composed of conservative components. But they also can include dissipative

40 components as well as some ports connected to external sources and through which energy can transit. These systems admit a power balance that can be decomposed into conservative, dissipative and source parts. They also can be simulated in such a way that the power balance (structured into conservative, dissipative and source parts) is preserved in the discrete-time domain, including 45 for nonlinear systems, see [14, p.32] and [15. These modeling and simulation 
tools are chosen, adapted and used to address the sound synthesis of the Fender Rhodes piano. Preliminary results have been presented in [16.

The paper is organized as follows. In section 1 the problem statement presents a simplified description of the Rhodes piano and sets the objectives.

50 Section 2 introduces the port-Hamiltonian $(\mathrm{pH})$ formulation. Section 3 is devoted to the physical modeling and the finite-dimensional $\mathrm{pH}$ formulation of elementary components. In particular, a finite-dimensional model of an EulerBernoulli cantilever beam is derived, based on a standard modal decomposition.

Then, in section 4, elementary components are connected, yielding the non-

55 linear finite-dimensional port-Hamiltonian system to simulate (step I). Section 5 details the numerical method that preserves the power balance (step II). Finally, in section 6, numerical results are presented and some signals are compared to a few measurements.

\section{Problem statement}

This section describes the Rhodes piano electromechanism. It focuses on the components that are selected to derive the physical model. Then, it states the scientific issues to be addressed to reach guaranteed-passive sound synthesis.

\subsection{Overview and main components}

A description of the Rhodes piano is given in figure 1. The complete system

65 is quite complex (38 components mentioned for each note in figure11@). The resonator is an asymmetric tuning fork (elements 7 to 13 and 19 in figure1b), where one of the prong is call the tine (element 13) and is struck by the hammer (elements 14 and 15), the other prong is called the tone bar (element 11). The constant magnetic induction field due to the pickup magnet in free air is mod-

70 ulated by the movement of the (magnetically conducting) tine, which results in a change of the magnetic flux in the coil, that generates an electromotive force (voltage) from Faraday's law of induction.

This paper focuses on the part depicted in figure 1 b), from the hammer (excited by a force) to the pickup (producing an output voltage). This part is 75 simplified and modeled by three multi-physical elements (c.f. figure 2):

1. a nonlinear hysteretic hammer $\mathrm{h}$, with adjustable material characteristics and geometry,

2. a linear damped cantilever beam $\mathrm{b}$, which can be tuned according to the shape and material properties,

80

3. a nonlinear pickup $\mathrm{p}$, the transverse and longitudinal position of which can be adjusted compared to the free-end beam extremity, which is connected to a $\mathrm{RC}$ analog filter (not represented in figure 2).

This simplification is justified or limited by the following considerations and assumptions. Elements 1-6 and 26-38 are excluded from figure 1 1 b. They are

85 the body or the key action mechanism. The body is assumed to be ideally rigid. The key action mechanism is ignored in the modeling. Indeed, the exhaust 


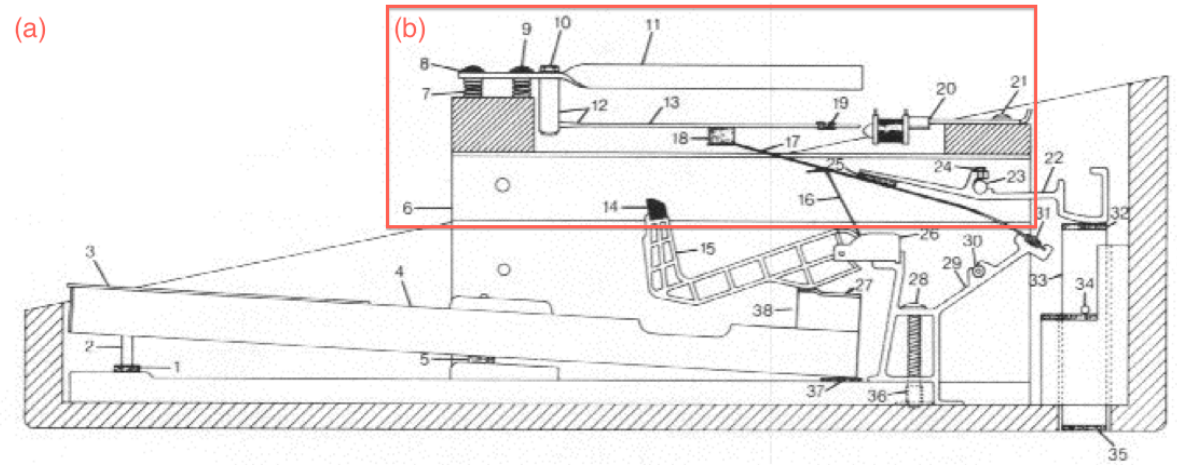

Figure 1: Overview of a single note of a Rhodes piano: (a) original schematics 17] (extracted from http://www.fenderrhodes.com courtesy of Frederik Adlers); (b) part selected for modeling.

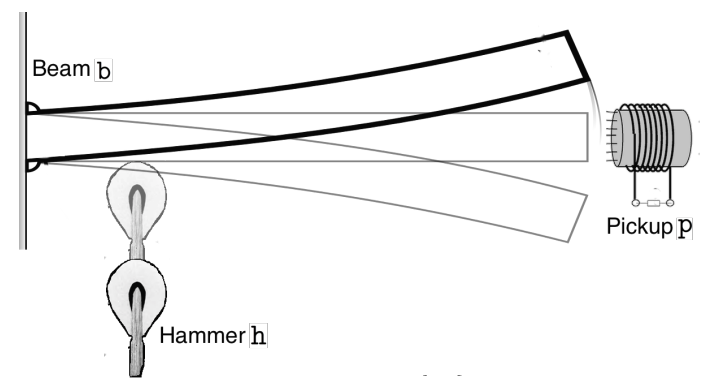

Figure 2: Schematic of the simplified electromechanical piano, with hammer $\mathrm{h}$, beam $\mathrm{b}$ and pickup p.

mechanism makes it out of contact with the hammer at the impact time. The hammer (elements 14-15) is then isolated and considered as the excitation part of the proposed model (see [18] for a study on a grand piano). The tuning fork module (elements 7-13 and 19) is the vibrating part of the system. Its modeling is simplified according to the following assumptions:

(A1) The cylindrical tine 13 is modeled as a linear damped Euler-Bernoulli beam.

(A2) The tone bar 11 is neglected, as well as its coupling with the tine through the solid pieces 10 and 12, on which the tine is clamped. Its main role is to store and restore mechanical energy to enhance the sustain. It also has an impact on the transient, the effect of which is not investigated here.

(A3) Elements 7-9 are composed of screws combined with springs with a high stiffness to adjust the transverse position of the tin with respect to the pickup. They are modeled as an ideally rigid connection with the body, located at an adjustable position. 
(A4) The fine tuning of the beam is addressed by adjusting the length of the beam rather than including the tuning mass (element 19).

These assumptions are motivated by the following reasons. The linear behavior assumed in (A1) has been validated in 19. Assumption (A2) is the most critical one in practice, as the tone bar pumps and restores mechanical energy to the tine. It has an impact on the transient and enhances the sustain. These effects are not investigated here in order to simplify the study. Assumptions (A3A4) correspond to the boundary conditions of a cantilever beam. This allows 110 an exact model order reduction through a decomposition on analytic eigenfunctions. The pickup and its placement (elements 20-21) are included in the modeling as they are known to have the main nonlinear impact on the output signal 20]. The damper module (elements $16-18$ and 22-25) is ignored in the modeling, meaning that the key release is not taken into account and that the beam is let free until the vibration extinction.

\subsection{Objectives}

The main objective of this work is to provide a physically-based sound synthesis that generates a large variety of realistic sounds. The second objective is to derive a simulation that preserves fundamental physical principles (causality, 120 stability and, more soundly, passivity and power balance) and that minimizes the computational load. To this end, the following approach is adopted: portHamiltonian formulation of the complete system, model order reduction of the infinite dimensional component (beam b), numerical method that preserves the power balance in the discrete-time domain, derivation of a $C_{++}$code from symbolic calculation to accelerate some costly operations.

\section{Port-Hamiltonian Systems}

This section starts from basic considerations on energy and passivity. It progressively introduces and recalls the port-Hamiltonian $(\mathrm{pH})$ formalism [10, 11, 12. It also presents an illustrative example. It is shown how this structure 130 guarantees the passivity of the model in the continuous time-domain.

\subsection{Considerations on energy and passivity}

Denote $\mathcal{E}(t) \geq 0$ the energy stored in an open physical system. If the system is autonomous and conservative, its time variation $\frac{d \mathcal{E}}{d t}(t)$ is zero. If the system is controlled (non-autonomous) and conservative, $\frac{\mathrm{d} \mathcal{E}}{\mathrm{d} t}(t)$ is the power $\mathcal{P}_{\text {ext }}(t)$ supplied by sources through external ports. For systems including dissipative phenomena with dissipated power $\mathcal{Q}(t) \geq 0$, the energy varies according to the power balance:

$$
\frac{\mathrm{d} \mathcal{E}}{\mathrm{d} t}(t)=\mathcal{P}_{\text {ext }}(t)-\mathcal{Q}(t)
$$

Such systems are passive in the sense that $\frac{d \mathcal{E}}{\mathrm{d} t} \leq \mathcal{P}_{\text {ext }}$. In particular, if the sources are not activated, $\frac{d \mathcal{E}}{d t} \leq 0$. The dynamic input-to-output behavior of such a system is the result of the power exchanges between isolated lumped or distributed 

(or can be a combination of): $N_{\mathcal{E}}$ components that store energy $\mathcal{E} \geq 0$ (moving mass, capacitors), $N_{\mathcal{Q}}$ components that dissipate power $\mathcal{Q} \geq 0$ (mechanical damping, transistors), $N_{\text {ext }}$ external ports that convey power $\mathcal{P}_{\text {ext }}(\in \mathbb{R})$ from sources (external forces, electrical batteries, etc) or any external system (active,

dist is between two sets of variables: flows $\mathbf{f}$ (velocities, currents, variations of magnetic flux, etc) and their associated efforts e (forces, voltages, magneto-motive forces, etc). All these quantities are defined in receiver convention, where $\mathcal{P}=\mathbf{f}^{\top} \mathbf{e}$ denotes the power received by the components.

145

The energy level of a storage component $n$ depends on an appropriate variable $x_{n}$ (the elongation $x_{n}=\ell$ for a spring, the charge $x_{n}=q$ for a capacitor, the magnetic flux $x_{n}=\phi$ for a coil, etc). This defines an energy function $\mathcal{H}_{n}$ $\left(\mathcal{H}_{n}(\ell)=\left(K \ell^{2}\right) / 2\right.$ for a linear spring with stiffness $K, \mathcal{H}_{n}(q)=q^{2} /(2 C)$ for a linear capacitor with capacitance $C, \mathcal{H}_{n}(\phi)=\phi^{2} /(2 L)$ for a linear coil with inductance $L$, etc). The total stored energy $\mathcal{E}$ is a function $\mathcal{H}$ of the state $\mathbf{x}=\left[x_{1}, \cdots, x_{N_{\mathcal{E}}}\right]^{\top}$ given by the sum $\mathcal{E}=\mathcal{H}(\mathbf{x})=\sum_{n=1}^{N_{\mathcal{E}}} \mathcal{H}_{n}\left(\mathbf{x}_{n}\right)$. The energy variation $\frac{\mathrm{d} \mathcal{E}}{\mathrm{d} t}$ in 11 is related to the state $\mathbf{x}$ with

$$
\frac{\mathrm{d} \mathcal{E}}{\mathrm{d} t}=\nabla \mathcal{H}(\mathbf{x})^{\top} \frac{\mathrm{d} \mathbf{x}}{\mathrm{d} t}
$$

This variation is precisely the received power, that is sum of the products of flows and efforts for all the components $\frac{d \mathcal{E}}{d t}=\mathbf{f}_{\mathcal{E}}^{\top} \mathbf{e}_{\mathcal{E}}$ with $\mathbf{f}_{\mathcal{E}}=\left[\mathrm{f}_{1}, \cdots, \mathrm{f}_{N_{\mathcal{E}}}\right]^{\top}$ and $\mathbf{e}_{\mathcal{E}}=\left[\mathrm{e}_{1}, \cdots, \mathrm{e}_{N_{\mathcal{E}}}\right]^{\top}$ (receiver convention). The external power supplied by sources is the sum of the products of flows and efforts localized at the ports (emitter convention), that is, $\mathcal{P}_{\text {ext }}=\mathbf{f}_{\text {ext }}^{\top} \mathbf{e}_{\text {ext }}$ with $\mathbf{f}_{\text {ext }}=\left[\mathrm{f}_{1}, \cdots, \mathrm{f}_{N_{\text {ext }}}\right]^{\top}$ and $\mathbf{e}_{\text {ext }}=\left[\mathrm{e}_{1}, \cdots, \mathrm{e}_{N_{\text {ext }}}\right]^{\top}$. These flows $\mathbf{f}_{\text {ext }}$ and efforts $\mathbf{e}_{\text {ext }}$ can be rearranged in two vectors, $\mathbf{u}$ (considered as a system input) and $\mathbf{y}$ (the associated output). This means that $\left(u_{n}, y_{n}\right)=\left(\mathbf{f}_{n}, \mathrm{e}_{n}\right)$ or $\left(u_{n}, y_{n}\right)=\left(\mathrm{e}_{n}, \mathrm{f}_{n}\right)$ for each pair in $\left(\mathbf{f}_{\text {ext }}, \mathbf{e}_{\text {ext }}\right)$ and yields

$$
\mathcal{P}_{\text {ext }}=\mathbf{u}^{\top} \mathbf{y}
$$

Finally, relating the flows and efforts of dissipative components (receiver convention) to the state and port variables, and expressing the total dissipated power as a sum of their product yield

$$
\mathcal{Q}(\mathbf{x}, \mathbf{u})=\left(\begin{array}{c}
\nabla \mathcal{H}(\mathbf{x}) \\
\mathbf{u}
\end{array}\right)^{\top} \mathbf{R}\left(\begin{array}{c}
\nabla \mathcal{H}(\mathbf{x}) \\
\mathbf{u}
\end{array}\right)
$$

the positivity of which is conditioned by that of matrix $\mathbf{R}$, which can depend on $\mathbf{x}$ (see section 2.3 for a simple example and sections 3 and 4 for the Rhodes piano).

\subsection{State-space representation of port-Hamiltonian systems}

The governing equations of a physical system relate the flows and the efforts introduced above. The port-Hamiltonian systems introduced in [10] (see also 
[11, 12, 21]) provide a formulation of such a class of equations for systems that fulfill the power balance (1) with (2), (3) and (4). A differential state-space representation is given by (see e.g. [12, eq 2.53]):

$$
\underbrace{\left(\begin{array}{c}
\frac{\mathrm{d} \mathbf{x}}{\mathrm{d} t} \\
-\mathbf{y}
\end{array}\right)}_{\mathbf{b}}=\underbrace{(\mathbf{J}(\mathbf{x})-\mathbf{R}(\mathbf{x}))}_{\mathbf{M}} \cdot \underbrace{\left(\begin{array}{c}
\nabla \mathcal{H}(\mathbf{x}) \\
\mathbf{u}
\end{array}\right)}_{\mathbf{a}}
$$

where $\mathcal{H}$ is the Hamiltonian, $\mathbf{J}$ is a skew-symmetric matrix $\left(\mathbf{J}^{\mathbf{T}}=-\mathbf{J}\right.$ for all $\mathbf{x}$ ) and $\mathbf{R}$ is a positive semidefinite matrix $\left(\mathbf{x}^{\top} \mathbf{R} \mathbf{x} \geq 0\right.$ for all $\left.\mathbf{x}\right)$. The system matrices are structured as follows:

$$
\mathbf{J}(\mathbf{x})=\left(\begin{array}{cc}
\mathbf{J}_{\mathbf{x}}(\mathbf{x}) & \mathbf{G}(\mathbf{x}) \\
-\mathbf{G}(\mathbf{x})^{\top} & \mathbf{J}_{\mathbf{y}}(\mathbf{x})
\end{array}\right), \mathbf{R}(\mathbf{x})=\left(\begin{array}{ll}
\mathbf{R}_{\mathbf{x}}(\mathbf{x}) & \mathbf{R}_{\mathbf{x y}}(\mathbf{x}) \\
\mathbf{R}_{\mathbf{x y}}(\mathbf{x})^{\top} & \mathbf{R}_{\mathbf{y}}(\mathbf{x})
\end{array}\right)
$$

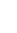

a in (3) and total dissipated power (4).

Proof. From the above definitions, we have $\mathbf{a} \mathbf{T} \mathbf{b}=\frac{\mathrm{d} \mathcal{E}}{\mathrm{d} t}-\mathcal{P}_{\text {ext }}$ and $\mathbf{a} \mathbf{T} \mathbf{b}=-\mathcal{Q}$ since $\mathbf{a}^{\top} \mathbf{M a}=-\left(\begin{array}{c}\nabla \mathcal{H}(\mathbf{x}) \\ \mathbf{u}\end{array}\right)^{\top} \mathbf{R}\left(\begin{array}{c}\nabla \mathcal{H}(\mathbf{x}) \\ \mathbf{u}\end{array}\right)$ from skew-symmetry of $\mathbf{J}$.

Remark 1 (Passivity, Lyapunov stability and numerical stability). A main interest of port-Hamiltonian systems is that they satisfy property 2.1. which ensures Lyapunov stability steming from passivity. In short, for a state space representation $\frac{\mathrm{d} \mathbf{x}}{\mathrm{d} t}=\mathbf{f}(\mathbf{x}, \mathbf{u}), \mathbf{y}=\mathbf{g}(\mathbf{x}, \mathbf{u})$ (with $\operatorname{dim}(\mathbf{u})=\operatorname{dim}(\mathbf{y})$ ) and storage function $\mathcal{H}$, passivity means that $\frac{\mathrm{d} \mathcal{H}(\mathbf{x})}{\mathrm{d} t} \leq \mathbf{y}^{\boldsymbol{\top}} \mathbf{u}=\mathcal{P}_{\text {ext }}$. Property 2.1 ensures this inequality. This implies that if the excitation stops $\left(\mathbf{u}=0\right.$ so that $\left.\mathcal{P}_{\text {ext }}=0\right)$, the storage function $\mathcal{H}$ stops increasing. More precisely, $\mathcal{E}=\mathcal{H}(\mathbf{x})$ is constant in the conservative case $(\mathcal{Q}=0)$ and it decreases in the dissipative case $(\mathcal{Q}>0)$. In these cases, the LaSalle invariance principle states that the trajectory $t \mapsto \mathbf{x}(t)$ tends toward the maximal invariant subspace $\mathbb{S}$ as $t \rightarrow+\infty$, with

$$
\mathbb{S}=\left\{\mathbf{x} \in \mathbb{R}^{N_{\mathcal{E}}} \text { s.t. } \nabla \mathcal{H}(\mathbf{x})^{\top} \mathbf{f}(\mathbf{x}, \mathbf{u}=0)=0\right\}
$$

Then, the Lyapunov theorem ensures the system stability as far as $\mathcal{H}$ is positive definite (see also [22, §4.1, 4.2 and 6.2] for details). If $\mathcal{H}$ is not definite, $\mathbb{S}$ provides the set of reachable asymptotic states. This property can be transfered to simulations, using the numerical method presented in section 5 that preserves the power balance in the discrete time domain. 


\subsection{Example}

In the sequel, for sake of readability, constant values are denoted by capital letters.

Consider the linear mass-spring-damper system depicted in figure 3 , where gravity is neglected. The mass position is denoted $q$, with $q=0$ the spring equilibrium. The system is excited by an external force $\left(f_{\text {ext }}\right)$.

This system includes $N_{\mathcal{E}}=2$ storage components, $N_{\text {ext }}=1$ external port and $N_{\mathcal{Q}}=1$ dissipative component. The storage components are: mass $M$ with momentum $x_{1}=\frac{\mathrm{d}(M q)}{\mathrm{d} t}$ and kinetic energy $\mathcal{H}_{1}\left(x_{1}\right)=x_{1}^{2} /(2 M)$, and spring $K$ with elongation $x_{2}=q$ and potential energy $\mathcal{H}_{2}\left(x_{2}\right)=\left(K x_{2}^{2}\right) / 2$. The ex-

175 ternal port is made of one input $\mathbf{u}=\mathrm{e}_{\text {ext }}=f_{\text {ext }}$ (external force) and one output $\mathbf{y}=\frac{\mathrm{d} q}{\mathrm{~d} t}$. The dissipative component is the damper with dissipated power $\mathcal{Q}_{A}(\mathbf{x})=H_{1}^{\prime}\left(x_{1}\right) A H_{1}^{\prime}\left(x_{1}\right)=A\left(\frac{\mathrm{d} q}{\mathrm{~d} t}\right)^{2}$. For the mass, $f_{M}=\frac{\mathrm{d} x_{1}}{\mathrm{~d} t}=M \frac{\mathrm{d}^{2} q}{\mathrm{~d} t^{2}}$ is the fictitious force (effort $\mathrm{e}_{1}$ ) and $\mathcal{H}_{1}^{\prime}\left(x_{1}\right)=\frac{\mathrm{d} q}{\mathrm{~d} t}$ is the velocity (flow $\mathrm{f}_{1}$ ). For the spring, $\frac{\mathrm{d} x_{2}}{\mathrm{~d} t}=\frac{\mathrm{d} q}{\mathrm{~d} t}$ is the velocity (flow $\mathrm{f}_{2}$ ) and $f_{K}=\mathcal{H}_{2}^{\prime}\left(x_{2}\right)=K q$ is the restoring force (effort $\mathrm{e}_{2}$ ). For the damper, the counteracting force is $f_{A}=A \frac{\mathrm{d} q}{\mathrm{~d} t}$ so that the associated effort is $\mathrm{e}_{A}=A \mathcal{H}_{1}^{\prime}\left(x_{1}\right)$.

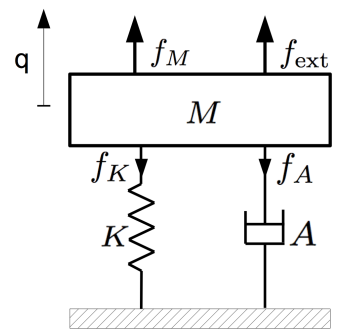

Figure 3: Damped harmonic oscillator with excitation.

Applying Newton's second law to this simple system yields

$$
\left(\begin{array}{c}
\mathrm{e}_{1} \\
\mathrm{f}_{2} \\
\hline-\mathrm{f}_{\mathrm{ext}}
\end{array}\right)=\left(\begin{array}{rr|r}
-A & -1 & +1 \\
+1 & 0 & 0 \\
\hline-1 & 0 & 0
\end{array}\right) \cdot\left(\begin{array}{c}
\mathrm{f}_{1} \\
\mathrm{e}_{2} \\
\hline \mathrm{e}_{\mathrm{ext}}
\end{array}\right) .
$$

From the constitutive laws of components, this equation exactly restores formulation $(5 \sqrt{6}$ ), block by block. This is summarized in table 1 .

\section{Models of components}

This section presents the multi-physical components that compose each note of a Rhodes piano: ( $\mathrm{h}$ ) a hammer in $\$ 3.1$. (b) a cantilever beam in $\$ 3.2$ and $(\mathrm{p})$ a pickup connected to a RC analog filter in $\S 3.3$. The hammer $\mathrm{h}$ is the nonlinear hysteretic model proposed in [23. This finite-dimensional system is associated with a non-quadratic energy and a nonlinear damping. The beam $\mathrm{b}$ is a linear 


\begin{tabular}{|c|c|}
\hline $\begin{array}{c}\text { State: } \\
\mathbf{x}=\left(M \frac{\mathrm{d} q}{\mathrm{~d} t}, q\right)^{\top}\end{array}$ & $\begin{array}{c}\text { Energy: } \\
\mathcal{H}(\mathbf{x})=\frac{x_{1}^{2}}{2 M}+\frac{K x_{2}^{2}}{2}\end{array}$ \\
\hline $\begin{array}{c}\text { Input: } \\
\mathbf{u}=f_{\text {ext }}\end{array}$ & $\begin{array}{l}\text { Output: } \\
\mathbf{y}=\frac{\mathrm{d} q}{\mathrm{~d} t}\end{array}$ \\
\hline $\mathbf{J}=\left(\begin{array}{rr|r}0 & -1 & +1 \\
+1 & 0 & 0 \\
\hline-1 & 0 & 0\end{array}\right), \mathbf{R}=\left(\begin{array}{rr|r}A & 0 & 0 \\
0 & 0 & 0 \\
\hline 0 & 0 & 0\end{array}\right)$ \\
\hline
\end{tabular}

Table 1: Port-Hamiltonian formulation $5 \sqrt{6}$ for the example 8 .

190 damped Euler-Bernoulli model [24, [25, §4.9]. A model order reduction of this infinite-dimensional model is derived, based on a standard modal decomposition. The pickup $\mathrm{p}$ is a finite-dimensional nonlinear model [26]. It is composed of one constant magnetic source (magnet), two linear components that stores energy (coil and capacitor), one linear resistor and one electrical port that delivers the output signal (voltage). Contrarily to the hammer, the nonlinearity of the pickup only comes from the magnetic flux induced in the coil by the movement of the beam extremity. The models of these three components prove passive so that they admit port-Hamiltonian formulations.

\subsection{Hammer $\mathrm{h}$}

The hammer is composed of a rigid core that is covered by a deformable felt. In this paper, its modeling is inspired from that proposed in [23] and used in e.g. [27, 28, 1] for the acoustic piano. This model describes nonlinear elastic phenomena (see e.g. 29, 30] for experimental validations) and nonlinear damping phenomena 31. This combination yields realistic nonlinear hysteretic effects that account for the shape memory of the felt.

Governing equations. Denote $q_{\mathrm{cont}}(t), q_{\mathrm{core}}(t)$ and $L_{\mathrm{h}}$, the position of the contact point, the top position of the core and the thickness of the felt at rest, respectively, in an inertial frame of reference (see figure 4). For the felt, the crushed thickness is 0 if $q_{\text {core }}(t)+L_{\mathrm{h}} \leq q_{\text {cont }}(t)$ and is

$$
q_{\mathrm{h}}(t)=q_{\mathrm{core}}(t)+L_{\mathrm{h}}-q_{\mathrm{cont}}(t)
$$

otherwise, so that the crushed rate is given by

$$
c_{\mathrm{h}}\left(q_{\mathrm{h}}\right)=\frac{\max \left(q_{\mathrm{h}}, 0\right)}{L_{\mathrm{h}}} .
$$


The elastic force $f_{\mathrm{h}}$ elast and the damping force $f_{\mathrm{h}}^{\text {damp }}$ due to the felt are nonlinear functions of the crush rate given by

$$
\begin{aligned}
& f_{\mathrm{h}}^{\text {elast }}\left(c_{\mathrm{h}}\right)=F_{\mathrm{h}} c_{\mathrm{h}} B_{\mathrm{h}}, \\
& f_{\mathrm{h}}^{\text {damp }}\left(c_{\mathrm{h}}\right)=A_{\mathrm{h}} L_{\mathrm{h}} \frac{\mathrm{d}\left(c_{\mathrm{h}} B_{\mathrm{h}}\right)}{\mathrm{d} t},
\end{aligned}
$$

where the inertial force of the felt is neglected and parameters are detailed in table 2. The hammer core can be considered as a mass $M_{\mathrm{h}}$ submitted to the felt force $-\left(f_{\mathrm{h}}^{\text {elast }}+f_{\mathrm{h}}^{\text {damp }}\right)$ and actuated by an external force $f_{\text {ext }}^{\text {core }}$ (see [18] $)$. Its governing equation is

$$
M_{\mathrm{h}} \frac{\mathrm{d}^{2} q_{\text {core }}}{\mathrm{d} t^{2}}=-f_{\mathrm{h}}^{\text {elast }}\left(c_{\mathrm{h}}\left(q_{\mathrm{h}}\right)\right)-f_{\mathrm{h}}^{\text {damp }}\left(c_{\mathrm{h}}\left(q_{\mathrm{h}}\right)\right)+f_{\text {ext }}^{\text {core }} .
$$

The mobile contact point with velocity $v_{\mathrm{ext}}^{\text {cont }}=\frac{\mathrm{d} q_{\text {cont }}}{\mathrm{d} t}$ experiences the reaction force $f_{\mathrm{h}}^{\text {elast }}+f_{\mathrm{h}}^{\text {damp }}$ so that the force exerted by the contact point on the felt is $f_{\text {ext }}^{\text {cont }}=-\left(f_{\mathrm{h}}^{\text {elast }}+f_{\mathrm{h}}^{\text {damp }}\right)$, and the mechanical power provided to the system is $P_{\text {cont }}=f_{\text {ext }}^{\text {cont }} v_{\mathrm{ext}}^{\text {cont }}$.

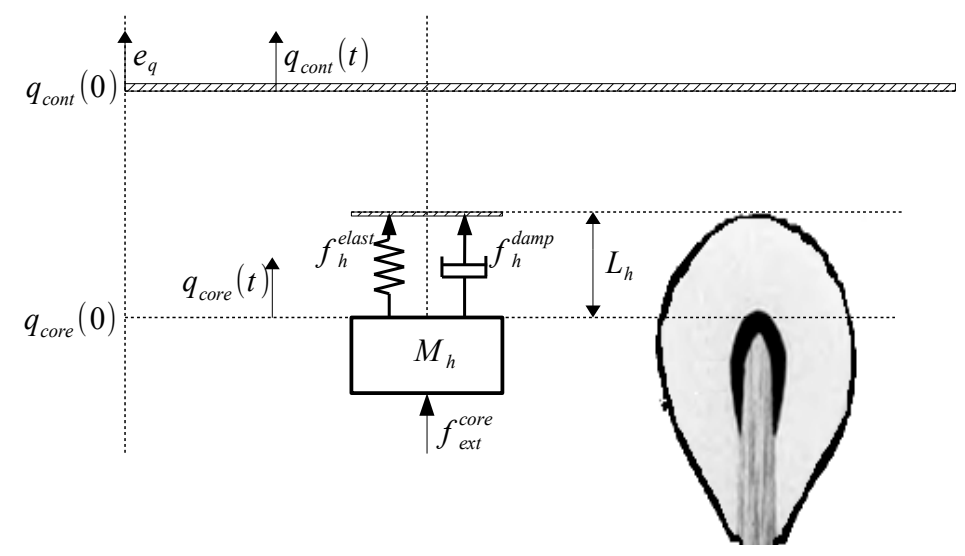

Figure 4: Mass-spring-damper model of the hammer and notations.

Port-Hamiltonian formulation. The port Hamiltonian system of the hammer is derived as in example 2.3 For the mass of the core, the state is the momentum $x_{\mathrm{h}, 1}=\frac{\mathrm{d} M_{\mathrm{h}} q_{\text {core }}}{\mathrm{d} t}$ with associated kinetic energy

$$
\mathcal{H}_{\mathrm{h}, 1}\left(x_{\mathrm{h}, 1}\right)=\frac{x_{\mathrm{h}, 1}^{2}}{2 M_{\mathrm{h}}} .
$$

For a spring, the potential energy is given by $\mathcal{H}_{\text {spring }}(q)=\int_{0}^{q} f_{\text {spring }}(\xi) \mathrm{d} \xi$. In example 2.3. the spring is symmetric and linear $\left(f_{\text {spring }}(q)=K q\right)$ so that $\mathcal{H}_{\text {spring }}(q)=K q^{2} / 2$ is quadratic. For the hammer felt, the spring is nonsymmetric (10) and nonlinear (11) so that the associated Hamiltonian $\mathcal{H}_{\mathrm{h}, 2}$ 


\begin{tabular}{rlll} 
Label & Description & Typical value & Unit \\
\hline \hline$M_{\mathrm{h}}$ & Total mass & $3 \times 10^{-2}$ & $\mathrm{Kg}$ \\
$L_{\mathrm{h}}$ & Felt thickness at rest & $15 \times 10^{-3}$ & $\mathrm{~m}$ \\
$B_{\mathrm{h}}$ & Felt characteristic exponent & 2.5 & Dimensionless \\
$F_{\mathrm{h}}$ & Elastic characteristic force & 13.8 & $\mathrm{~N}$ \\
$A_{\mathrm{h}}$ & Felt damping coefficient & 0.184 & N.s.m \\
\hline
\end{tabular}

Table 2: Physical parameters for the hammer $\mathrm{h}$. These parameters correspond to typical values found in [30, 23] or [32, (I.2.2)] with formula $f_{\mathrm{h}}^{\text {elast }} \equiv K e^{p}, f_{\mathrm{h}}^{\text {damp }} \equiv R \frac{\mathrm{d}\left(e^{p}\right)}{\mathrm{d} t}$ and $e=L_{\mathrm{h}} C_{\mathrm{h}}$, that is, $p=B_{\mathrm{h}}=2.5, K=L_{\mathrm{h}}^{-B_{\mathrm{h}}} F_{\mathrm{h}}=5 \times 10^{5} \mathrm{~N} \cdot \mathrm{m}^{-2.5}$ and $R=L_{\mathrm{h}}^{1-B_{\mathrm{h}}} A_{\mathrm{h}}=100$ N.s.m ${ }^{-2.5}$.

is a non-even and non-quadratic function of the state $x_{\mathrm{h}, 2}=q_{\mathrm{h}}$ :

$$
\mathcal{H}_{\mathrm{h}, 2}\left(x_{\mathrm{h}, 2}\right)=\frac{L_{\mathrm{h}} F_{\mathrm{h}}}{B_{\mathrm{h}}+1} c_{\mathrm{h}}\left(x_{\mathrm{h}, 2}\right)^{B_{\mathrm{h}}+1} .
$$

The flows and efforts of the system are derived as follows. For the mass, $\frac{\mathrm{d} x_{\mathrm{h}, 1}}{\mathrm{~d} t}=\frac{\mathrm{d}^{2}\left(M_{\mathrm{h}} q_{\mathrm{core}}\right)}{\mathrm{d} t^{2}}$ is the inertial force (effort labeled $\left.\mathrm{e}_{\mathrm{h}, 1}\right)$ and $\mathcal{H}_{\mathrm{h}, 1}^{\prime}\left(x_{\mathrm{h}, 1}\right)=\frac{\mathrm{d}}{\mathrm{d} t} q_{\text {core }}$ is the velocity (flow labeled $f_{h, 1}$ ). For the spring, $\frac{d x_{h, 2}}{d t}=\frac{d q_{h}}{d t}$ is homogeneous to a velocity (flow $\left.\mathrm{f}_{\mathrm{h}, 2}\right)$ and $\mathcal{H}_{2}^{\prime}\left(x_{\mathrm{h}, 2}\right)=f_{\mathrm{h}}{ }^{\text {elast }}\left(c_{\mathrm{h}}\left(q_{\mathrm{h}}\right)\right.$ ) is the spring force (effort $\left.\mathrm{e}_{\mathrm{h}, 2}\right)$. For the damper, the damping force is $f_{\mathrm{h}}^{\text {damp }}\left(q_{\mathrm{h}}\right)=\frac{A_{\mathrm{h}} L_{\mathrm{h}}}{B_{\mathrm{h}}} c_{\mathrm{h}}\left(q_{\mathrm{h}}\right)^{B_{\mathrm{h}}-1} \frac{\mathrm{d} q_{\mathrm{h}}}{\mathrm{d} t}$ so that the effort is $\mathrm{e}^{\mathrm{damp}}=r_{\mathrm{h}}\left(x_{\mathrm{h}, 2}\right)\left(\mathcal{H}_{\mathrm{h}, 1}^{\prime}\left(x_{\mathrm{h}, 1}\right)-v_{\mathrm{ext}}^{\text {cont }}\right)$ with

$$
r_{\mathrm{h}}\left(q_{\mathrm{h}}\right)=\frac{A_{\mathrm{h}} L_{\mathrm{h}}}{B_{\mathrm{h}}} c_{\mathrm{h}}\left(q_{\mathrm{h}}\right)^{B_{\mathrm{h}}-1} .
$$

The $\mathrm{pH}$ system of the hammer has two external ports. One is associated with the core velocity $\frac{\mathrm{d} q_{\text {core }}}{\mathrm{d} t}\left(\right.$ flow $\left.\mathrm{f}_{\mathrm{h}, 3}=\mathrm{f}_{\mathrm{h}, 1}\right)$ and the associated external force $f_{\text {ext }}^{\text {core }}$ (effort $\mathrm{e}_{\mathrm{h}, 3}$ ). The other one is associated with the contact point velocity $v_{\mathrm{ext}}^{\text {cont }}$ (flow $\mathrm{f}_{\mathrm{h}, 4}$ ) and the associated external force $f_{\text {ext }}^{\text {cont }}$ (effort $\mathrm{e}_{\mathrm{h}, 4}=-\mathrm{e}_{\mathrm{h}, 2}-\mathrm{e}^{\mathrm{damp}}$ so that $f_{h, 4} e_{h, 4}$ is the power provided by the contact point to the system). Inputs and outputs are chosen to be $\mathbf{u}_{\mathrm{h}}=\left(\mathrm{e}_{\mathrm{h}, 3}, \mathrm{f}_{\mathrm{h}, 4}\right), \mathbf{y}_{\mathrm{h}}=\left(\mathrm{f}_{\mathrm{h}, 3}, \mathrm{e}_{\mathrm{h}, 4}\right)$. This choice restores the governing equation $(12)$, summarized in table 3 .

\subsection{Beam $\mathrm{b}$}

In this paper, the standard Euler-Bernoulli modeling of a damped cantilever beam is used, which results in a linear partial-differential equation (see e.g. [24]). To cope with numerical realizability, we firstly apply a standard modal decomposition and recast the resulting set of ordinary differential equations as a finite dimensional $\mathrm{pH}$ system (see [33] and particularly [34, § 1.3$]$ for infinite dimensional $\mathrm{pH}$ description). This contrasts with the finite-element spatial discretization [35] considered in e.g. [1, 2, 3, 4. 


\begin{tabular}{|c|c|}
\hline $\begin{array}{c}\text { State: } \\
\mathbf{x}_{\mathrm{h}}=\left(\frac{\mathrm{d}}{\mathrm{d} t}\left(M_{\mathrm{h}} q_{\text {core }}\right), q_{\mathrm{h}}\right)^{\top}\end{array}$ & $\begin{array}{c}\text { Energy: } \\
\mathcal{H}_{\mathrm{h}}\left(\mathbf{x}_{\mathrm{h}}\right)=\mathcal{H}_{\mathrm{h}, 1}\left(x_{\mathrm{h}, 1}\right)+\mathcal{H}_{\mathrm{h}, 2}\left(x_{\mathrm{h}, 2}\right)\end{array}$ \\
\hline $\begin{array}{c}\text { Input: } \\
\mathbf{u}_{\mathrm{h}}=\left(f_{\text {ext }}^{\text {core }}, v_{\text {ext }}^{\text {cont }}\right)^{\top}\end{array}$ & $\begin{array}{c}\text { Output: } \\
\mathbf{y}_{\mathrm{h}}=\left(\frac{\mathrm{d}}{\mathrm{d} t} q_{\text {core }}, f_{\text {ext }}^{\text {cont }}\right)^{\top}\end{array}$ \\
$\mathbf{J}=\left(\begin{array}{rr|rr}0 & -1 & +1 & 0 \\
+1 & 0 & 0 & -1 \\
\hline-1 & 0 & 0 & 0 \\
0 & +1 & 0 & 0\end{array}\right), \mathbf{R}=\left(\begin{array}{cc|cc}+r_{\mathrm{h}}\left(x_{\mathrm{h}, 2}\right) & 0 & 0 & -r_{\mathrm{h}}\left(x_{\mathrm{h}, 2}\right) \\
0 & 0 & 0 & 0 \\
\hline 0 & 0 & 0 & 0 \\
-r_{\mathrm{h}}\left(x_{\mathrm{h}, 2}\right) & 0 & 0 & +r_{\mathrm{h}}\left(x_{\mathrm{h}, 2}\right)\end{array}\right)$. \\
\hline
\end{tabular}

Table 3: Port-Hamiltonian formulation 5,6 for the hammer (see 90 and $13-15$ for definitions). In this case, the invariant subspace (7) is described by (13)14 with (10), that is, $\mathbb{S}_{\mathrm{h}}=\left\{\mathbf{x}_{\mathrm{h}} \in \mathbb{R}^{2}\right.$ s.t. $\left.\left[\mathbf{x}_{\mathrm{h}}\right]_{1}=0,\left[\mathbf{x}_{\mathrm{h}}\right]_{2} \leq 0\right\}$. Inequality $\left[\mathbf{x}_{\mathrm{h}}\right]_{2} \leq 0$ is due to the fact that the hammer position goes to minus infinity with constant velocity $\left(\left[\mathbf{x}_{\mathrm{h}}\right]_{1}=0\right)$, after it has rebounded on the contact point.

Euler-Bernoulli modeling. Denoting $z \in\left(0, L_{\mathrm{b}}\right)$ the spatial coordinate along the beam, the Euler-Bernoulli modeling [24, [25, §4.9] of transverse deflect $q(z, t)$ is

$$
M_{\mathrm{b}} \partial_{t}^{2} q+A_{\mathrm{b}} \partial_{t} q+K_{\mathrm{b}} \partial_{z}^{4} q=f_{\mathrm{b}}
$$

where parameters are detailed in table 4 . Initial conditions are $q(z, 0)=0$ and $\partial_{t} q(z, 0)=0$. The configuration space of the deflect $q$ is a Hilbert space $\mathbb{H}=L^{2}\left(0, L_{\mathrm{b}}\right)$. The bounded inertia operator and damping operator associated with (16) are $\mathcal{M}=M_{\mathrm{b}} \mathcal{I}$ and $\mathcal{C}=A_{\mathrm{b}} \mathcal{I}$, respectively, where $\mathcal{I}$ denotes the identity operator. The stiffness operator is $\mathcal{K} q=K_{\mathrm{b}} \partial_{z}^{4} q$ with domain $\mathcal{D}(\mathcal{K})=$ $\left\{q \in H^{4}\left(0, L_{\mathrm{b}}\right)\right.$ s.t. $\left.q(0)=0, \partial_{z} q(0)=0, \partial_{z}^{2} q\left(L_{\mathrm{b}}\right)=0, \partial_{z}^{3} q\left(L_{\mathrm{b}}\right)=0\right\}\left(H^{4}\right.$ denotes the standard Sobolev space), that includes the boundary conditions for a cantilever beam, namely: (i) no displacement at the base $q(0, t)=0$, (ii) no bending at the base $\partial_{z} q(0, t)=0$, (iii) no bending moment at the free end $\partial_{z}^{2} q(1, t)=0$, (iv) no shearing force acting at the free end $\partial_{z}^{3} q(1, t)=0$. Operator $\mathcal{K}$ fulfills standard well-posedness properties such as being densely defined, closed, unbounded and self-adjoint on the Hilbert space $\mathbb{H}$ (see [36, §2] for technical details, [25, §4.9] for the details on the cantilever beam and [37, §4 and 5] for a complete functional setting of the Euler-Bernouilli modeling with free ends in the port-Hamiltonian formalism). The total mechanical energy is [38, §7]:

$$
\mathcal{E}_{\mathrm{b}}=\frac{1}{2} \int_{0}^{L_{\mathrm{b}}}\left(K_{\mathrm{b}}\left(\partial_{z}^{2} q\right)^{2}+M_{\mathrm{b}}\left(\frac{\mathrm{d} q}{\mathrm{~d} t}\right)^{2}\right) \mathrm{d} z .
$$

Finite dimensional approximation. The linear boundary value problem (16) admits an orthogonal basis of eigenfunctions $\mathrm{B}=\left\{\psi_{m}\right\}_{m \in \mathbb{N}_{*}}$ on the Hilbert space 


\begin{tabular}{llll} 
Label & Description & Typical value & Unit \\
\hline \hline$M_{\text {steel }}$ & Mass density & 7750 & $\mathrm{~kg} \cdot \mathrm{m}^{-3}$ \\
$E_{\text {steel }}$ & Young Modulus & $180.10^{9}$ & $\mathrm{~N} \cdot \mathrm{m}^{-2}$ \\
$R_{\mathrm{b}}$ & Radius & $10^{-3}$ & $\mathrm{~m}$ \\
$A_{\mathrm{b}}$ & Damping coefficient & $5.10^{-2}$ & $\mathrm{~N} \cdot \mathrm{s}^{-1}$ \\
$F_{\mathrm{b}}$ & Tuning frequency & 440 & $\mathrm{~Hz}$ \\
\hline$M_{\mathrm{b}}$ & Mass per unit length & $\pi R_{\mathrm{b}}^{2} M_{\text {steel }}$ & $\mathrm{kg} \cdot \mathrm{m}^{-1}$ \\
$I_{\mathrm{b}}$ & Moment of inertia & $\frac{\pi}{4} R_{\mathrm{b}}^{4}$ & $\mathrm{~m}$ \\
$K_{\mathrm{b}}$ & Flexural rigidity & $E_{\text {steel }} I_{\mathrm{b}}$ & $\mathrm{N} \cdot \mathrm{m}^{2}$ \\
$L_{\mathrm{b}}$ & Length & $5.54 .10^{-2}$ & $\mathrm{~m}$ \\
\hline
\end{tabular}

Table 4: Fixed and deduced physical parameters for a cylindrical beam $\mathrm{b}$. The moment of inertia of the cross section is given for a cylindrical shape. The length for the desired tone $\omega_{\mathrm{b}}=2 \pi F_{\mathrm{b}}$ is obtain from a numerical evaluation of 18$)$ with $\kappa_{m}=\sqrt[4]{\omega_{m}^{2} M_{\mathrm{b}} / K_{\mathrm{b}}}$ the dispersion relation.

$\mathbb{H}$. Functions $\psi_{m}$ are the spatial modes, detailed in AppendixA.1. They satisfy the boundary conditions (i-iv), $\partial_{z}^{4} \psi(z)=\kappa^{4} \psi(z)$ for appropriate wave number $\kappa$ and $\left\langle\psi_{m}, \psi_{p}\right\rangle=\delta_{m, p}$ (Kronecker's symbol) for all $(m, p) \in \mathbb{N}_{*}^{2}$, where the scalar product on $\mathbb{H}$ is defined by $\langle f, g\rangle=\int_{0}^{L_{\mathrm{b}}} f(z) g(z) \mathrm{d} z$. Wave numbers $\kappa_{m}$ are the solutions of

$$
\cosh \left(\kappa_{m} L_{\mathrm{b}}\right) \cos \left(\kappa_{m} L_{\mathrm{b}}\right)+1=0,
$$

with increasing modulus with respect to the index $m \in \mathbb{N}^{*}$.

The force $f_{\mathrm{b}}^{\text {ext }}$ is distributed according to $\sigma_{\mathrm{h}}(z)=\frac{1}{D_{\mathrm{h}}} \mathbf{1}\left(z-z_{\mathrm{h}}\right)_{\left[-D_{\mathrm{h}} / 2,+D_{\mathrm{h}} / 2\right]}$ and excites all the eigenmodes of the linear model. For the sound synthesis, we are interested by the eigenfrequencies $\nu_{m}$ below the Shannon frequency $\frac{1}{2 T}$ (with $T$ the sampling period). Hence, we select the $M$ first modes such that $\nu_{M}<\frac{1}{2 T}<\nu_{M+1}$. Then, we introduce $\mathbf{f}=\left(f_{1}, \ldots, f_{M}\right)^{\top}=\Omega f_{\mathrm{b}}^{\text {ext }}$ with $\Omega=\left\langle\sigma_{\mathrm{h}}, \Psi\right\rangle$ for $\Psi=\left(\psi_{1}, \ldots, \psi_{M}\right)^{\top}$ (see AppendixA.2). The relations satisfied by the modal displacements $\mathbf{q}_{\mathrm{b}}=\langle q, \Psi\rangle$ are obtained by projecting equation (16) on truncated basis $\mathrm{B}_{M}=\left\{\psi_{m}\right\}_{1 \leq m \leq M}$. This yields the following ordinary differential equations:

$$
M_{\mathrm{b}} \frac{\mathrm{d}^{2} \mathbf{q}_{\mathrm{b}}}{\mathrm{d} t^{2}}+A_{\mathrm{b}} \frac{\mathrm{d} \mathbf{q}_{\mathrm{b}}}{\mathrm{d} t}+K_{\mathrm{b}} \mathbf{L} \mathbf{q}_{\mathrm{b}}=\mathbf{f}=\Omega f_{\mathrm{b}}^{\text {ext }},
$$

with $\mathbf{L}=\operatorname{diag}\left(\kappa_{1}^{4}, \ldots, \kappa_{M}^{4}\right)$, which rewrites $\frac{\mathrm{dx}_{\mathrm{b}}}{\mathrm{d} t}=\mathbf{A} \mathbf{x}_{\mathrm{b}}+\mathbf{B} \mathbf{u}_{\mathrm{b}}$ with input $\mathbf{u}_{\mathrm{b}}=f_{\mathrm{b}}^{\text {ext }}$, state $\mathbf{x}_{\mathrm{b}}=\left(\mathbf{q}_{\mathrm{b}}^{\top}, M_{\mathrm{b}} \frac{\mathrm{d} \mathbf{q}_{\mathrm{b}} \mathbf{d} t}{\mathrm{~d}}\right)^{\top}, \mathbf{B}=\left(\mathbb{O}, \Omega^{\top}\right)^{\top}$ and

$$
\mathbf{A}=\left(\begin{array}{cc}
0 & \frac{1}{M_{\mathrm{b}}} \mathbf{I}_{\mathbf{d}} \\
-K_{\mathrm{b}} \mathbf{L} & -\frac{A_{\mathrm{b}}}{M_{\mathrm{b}}} \mathbf{I}_{\mathbf{d}}
\end{array}\right),
$$

where $\mathbb{O}$ denotes the null matrix and $\mathbf{I}_{\mathbf{d}}$ denotes the identity matrix. 
Port-Hamiltonian formulation. The power provided by the external force $f_{\mathrm{b}}^{\text {ext }}$ is the product of the later with the collocated velocity $v_{\mathrm{b}}^{\text {ext }}$ reconstructed from the modal velocities $v_{\mathrm{b}}^{\text {ext }}=\Omega^{\top} \frac{\mathrm{d} \mathbf{q}_{\mathrm{b}}}{\mathrm{d} t}$. This yields to select the output $\mathbf{y}_{\mathrm{b}}=v_{\mathrm{b}}^{\text {ext }}=\mathbf{B}^{\top} \nabla \mathcal{H}_{\mathrm{b}}$ so that the incoming power is $\mathcal{P}=\mathbf{y}_{\mathrm{b}}^{\top} \mathbf{u}_{\mathrm{b}}=\int_{0}^{L_{\mathrm{b}}} \mathbf{y}_{\mathrm{b}}^{\top} \Psi \Psi^{T} \mathbf{u}_{\mathrm{b}} \mathrm{d} z$ (with $\mathbf{u}_{\mathrm{b}}=f_{\mathrm{b}}^{\text {ext }}$ ). From (17) and the modal reconstruction $q=\mathbf{q}_{\mathrm{b}}^{\top} \Psi$, the total energy of the beam is the Hamiltonian $\mathcal{H}_{\mathrm{b}}\left(\mathbf{x}_{\mathrm{b}}\right)=\frac{1}{2} \mathbf{x}_{\mathrm{b}}^{T} \mathbf{W} \mathbf{x}_{\mathrm{b}}$, with

$$
\mathbf{W}=\left(\begin{array}{cc}
K_{\mathrm{b}} \mathbf{L} & \mathbb{0} \\
\mathbb{0} & M_{\mathrm{b}}{ }^{-1} \mathbf{I}_{\mathbf{d}}
\end{array}\right) .
$$

The resulting port-Hamiltonian system is given in table 5 .

\begin{tabular}{|c|c|}
\hline $\begin{array}{c}\text { State: } \\
\mathbf{x}_{\mathrm{b}}=\left(\mathbf{q}_{\mathrm{b}}, M_{\mathrm{b}} \frac{\mathrm{d} \mathbf{q}_{\mathrm{b}}}{\mathrm{d} t}\right)^{\top}\end{array}$ & $\begin{array}{c}\text { Energy: } \\
\mathcal{H}_{\mathrm{b}}\left(\mathbf{x}_{\mathrm{b}}\right)=\frac{1}{2} \mathbf{x}_{\mathrm{b}}^{\top} \mathbf{W} \mathbf{x}_{\mathrm{b}}\end{array}$ \\
\hline $\begin{array}{c}\text { Input: } \\
\mathbf{u}_{\mathrm{b}}=f_{\mathrm{b}}^{\text {ext }}\end{array}$ & $\begin{array}{c}\text { Output: } \\
\mathbf{y}_{\mathrm{b}}=v_{\mathrm{b}}^{\text {ext }}\end{array}$ \\
\hline $\mathbf{J}=\left(\begin{array}{cc|c}\mathbb{0} & \mathbf{I}_{\mathbf{d}} & \mathbb{0} \\
-\mathbf{I}_{\mathbf{d}} & \mathbb{0} & \Omega \\
\hline \mathbb{0} & -\Omega^{\top} & \mathbb{0}\end{array}\right), \mathbf{R}=\left(\begin{array}{cc|c}\mathbb{0} & \mathbb{0} & \mathbb{0} \\
\mathbb{0} & A_{\mathrm{b}} \mathbf{I}_{\mathbf{d}} & \mathbb{0} \\
\hline \mathbb{0} & \mathbb{0} & \mathbb{0}\end{array}\right)$ \\
\end{tabular}

Table 5: Port-Hamiltonian formulation 5 for the Euler-Bernoulli beam (see 20 for definition of $\mathbf{W})$. In this case, the invariant subspace $\left[7\right.$ is $\mathbb{S}_{\mathrm{b}}=\{\mathbb{O}\} \subset \mathbb{R}^{2 M}$.

\subsection{Pickup $\mathrm{p}$}

The electromagnetic pickup p includes a magnet (source of constant magnetic field $B_{\mathrm{p}}^{\mathrm{mag}}$ ) and a coil with $N_{\text {coil }}$ wire turns (sensor for the magnetic field variation). In this paper, the physical modeling of electric guitar pickups [26] is adapted to the system depicted in figure 6 (see also the physical model in [39, and physically inspired signal processing modules in [40, 41]). In short, a piece of magnetic material (here the beam) changes the spatial distribution of the magnetic field due to the magnet only, hence a change to the flux in the coil $\phi_{\mathrm{p}}$. Additionally, the coil is connected to a linear RC circuit, so that its total flux is $\phi_{\text {coil }}=\phi_{\mathrm{p}}+\phi_{\mathrm{RC}}$ with $\frac{\mathrm{d} \phi_{\mathrm{RC}}}{\mathrm{d} t}=\frac{v_{\text {coil }}}{N_{\text {coil }}}$ (Faraday's law of induction with coil voltage $\left.v_{\text {coil }}\right)$.

First, the coil is modeled as a magnetic capacity in the magnetic domain according to the gyrator-capacitor approach [42, 43]. Second, we recall the main steps in the derivation of the flux $\phi_{\mathrm{p}}$ from [26] for the system in figure 6 . Third, the coil is connected to the $\mathrm{RC}$ circuit and the resulting model is recast 250 as a port-Hamiltonian system. 
Magneto-electric transducer. According to the gyrator-capacitor approach detailed in [42, 43, we adopt the magnetic flux variation $\frac{\mathrm{d} \phi}{\mathrm{d} t}$ and the magnetomotive force $h$ as magnetic flow and effort variables, respectively $\left(\frac{\mathrm{d} \phi}{\mathrm{d} t} h \equiv \mathcal{P}\right)$. This permits to model the coil as a linear magnetic capacity: $x_{\text {coil }}=\phi_{\text {coil }}$ and $h_{\text {coil }}=\mathcal{H}_{\text {coil }}^{\prime}\left(x_{\text {coil }}\right)$ with $\mathcal{H}_{\text {coil }}\left(x_{\text {coil }}\right)=\frac{1}{2 C_{\text {coil }}} x_{\text {coil }}^{2}$. The connection to the electrical domain with $v_{\text {coil }}$ the tension and $i_{\text {coil }}$ the current trough the coil is obtained from the combination of Ampere's theorem $\left(h_{\text {coil }}=N_{\text {coil }} i_{\text {coil }}\right)$ and Faraday's law $\left(v_{\text {coil }}=N_{\text {coil }} \frac{\mathrm{d} \phi_{\text {coil }}}{\mathrm{d} t}\right)$, yielding a magneto-electric gyrator with ratio $\frac{1}{N_{\text {coil }}}$ :

$$
\left(\begin{array}{c}
\frac{\mathrm{d} \phi_{\text {coil }}}{\mathrm{d} t} \equiv \frac{\mathrm{d} x_{\text {coil }}}{\mathrm{d} t} \\
-i_{\text {coil }}
\end{array}\right)=\left(\begin{array}{cc}
0 & \frac{1}{N_{\text {coil }}} \\
-\frac{1}{N_{\text {coil }}} & 0
\end{array}\right)\left(\begin{array}{c}
h_{\text {coil }} \equiv \mathcal{H}_{\text {coil }}^{\prime}\left(x_{\text {coil }}\right) \\
v_{\text {coil }}
\end{array}\right) \text {. }
$$

Notice this connection is conservative: $v_{\text {coil }} i_{\text {coil }}=h_{\text {coil }} \frac{\mathrm{d} \phi_{\text {coil }}}{\mathrm{d} t}$.

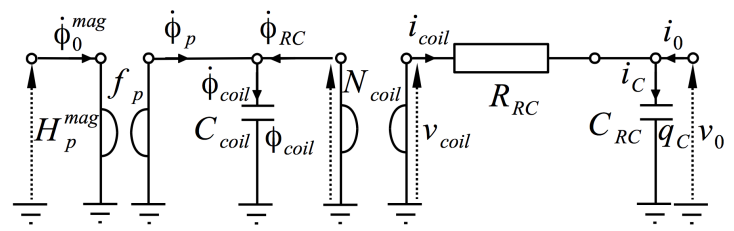

Figure 5: Schematic of the pickup connected to the RC circuit, with $h_{0}$ the constant magnetomotive force due to the magnet, $f_{\mathrm{p}}$ as in equation 25 and $L$ the magnetic capacitance of the coil with $N_{\text {coil }}$ wire turns. The input current is $i_{0}=0$ (high resistive load).

\begin{tabular}{llll} 
Label & Description & Typical value & Unit \\
\hline \hline$B_{\mathrm{p}}$ & Magnetic field of the magnet & $\pi 4.10^{-7}$ & $\mathrm{~T}$ \\
$U_{0}$ & Magnetic permeability of vacuum & $\pi 4.10^{-7}$ & $\mathrm{H} . \mathrm{m}^{1}$ \\
$U_{\text {steel }}$ & Magnetic permeability of steel & $5.10^{-3}$ & $\mathrm{H} . \mathrm{m}^{1}$ \\
$C_{\text {coil }}$ & Magnetic capacity & $3.07 .10^{-5}$ & $\mathrm{H}$ \\
$R_{\mathrm{p}}$ & Coil radius & $5.10^{-3}$ & $\mathrm{~m}$ \\
$L_{\text {hor }}$ & Horizontal decay & $10^{-2}$ & $\mathrm{~m}$ \\
$L_{\text {ver }}$ & Vertical decay & $10^{-3}$ & $\mathrm{~m}$ \\
$N_{\text {coil }}$ & Number of wire turns & 100 & Dimensionless \\
$R_{\mathrm{RC}}$ & Electrical resistance & $10^{3}$ & $\Omega$ \\
$C_{\mathrm{RC}}$ & Electrical capacity & $33.10^{-8}$ & $F$ \\
\hline$U_{\text {rel }}$ & Relative magnetic permeability of steel & $\frac{U_{\text {steel }}}{U_{0}}$ & Dimensionless \\
\hline
\end{tabular}

Table 6: Physical parameters for the pickup p.

Mechano-magnetic transducer. The two dimensional modeling proposed in [26] for the electric guitar pickup is recalled thereafter. A vibrating sphere with radius $A_{\mathrm{b}}$ (same as the beam) is immersed in a constant magnetic induction 255 field $B_{\mathrm{p}}^{\text {mag }}$ due to the magnet. Since there is no conducting current, the total magnetic excitation field is irrotational and derives from a magnetic potential. 


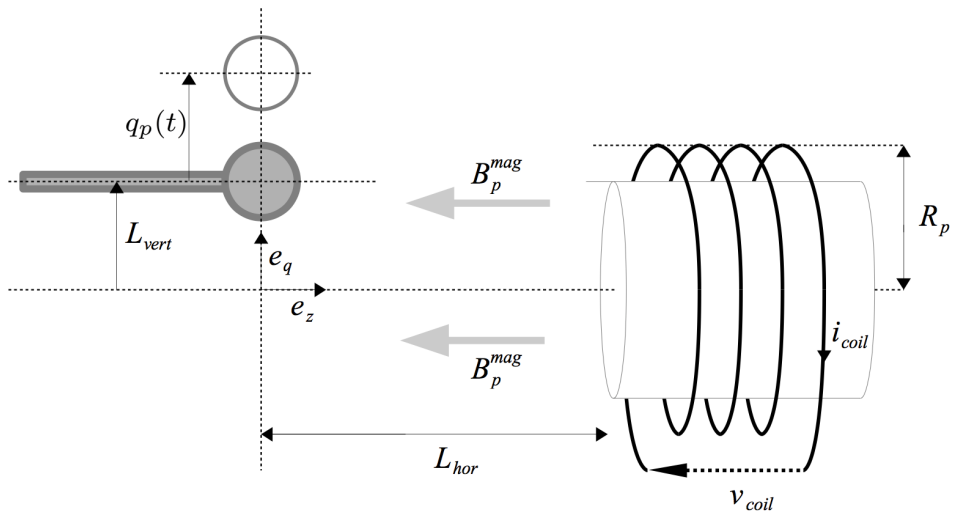

Figure 6: Description of the pickup. $q_{p}(t)$ is the position of the end of the beam measured from its rest position, $L_{\mathrm{ver}}$ is a vertical decay, $q_{c}$ is the position of the center of the magnet from the origin of the frame associated with the beam, $L_{\mathrm{hor}}$ is the distance between the coil and the beam, $R_{\mathrm{p}}=\frac{a_{c}^{2}}{2}$ is the radius of the coil.

The magnetization of the sphere with relative magnetic permeability $U_{\text {rel }}$ results in an additional term to the potential due to the magnet only. The integration of the total magnetic field over the area bounded by a single wire turn yields the following magnetic flux (see coordinate system and description in figure 6):

$$
\begin{aligned}
\phi_{\mathrm{p}} & =4 B_{\mathrm{p}}^{\mathrm{mag}} R_{\mathrm{p}}^{2}\left(4 R_{\mathrm{p}}^{3} \Delta_{U}\left(f_{\phi}\left(-\left(q_{\mathrm{p}}+L_{\mathrm{ver}}\right)\right)+f_{\phi}\left(q_{\mathrm{p}}+L_{\mathrm{ver}}\right)\right)-1\right) \\
f_{\phi}\left(q_{\mathrm{p}}\right) & =\frac{q_{\mathrm{p}}+R_{\mathrm{p}}}{L_{\text {hor }}^{2}+\left(q_{\mathrm{p}}+R_{\mathrm{p}}\right)^{2}},
\end{aligned}
$$

where $q_{\mathrm{p}}(t)$ is the vertical displacement measured from rest position defined as $\left(L_{\mathrm{hor}}, L_{\mathrm{ver}}\right)$ and $\Delta_{U}=\frac{U_{\mathrm{rel}}-1}{U_{\mathrm{rel}}+1}$. The induced tension depends on the flux variation, which reads

$$
\begin{aligned}
\frac{\mathrm{d} \phi_{\mathrm{p}}}{\mathrm{d} t} & =f_{\mathrm{p}}\left(q_{\mathrm{p}}, \frac{\mathrm{d} q_{\mathrm{p}}}{\mathrm{d} t}\right) H_{\mathrm{p}}^{\operatorname{mag}} \\
f_{\mathrm{p}}\left(q_{\mathrm{p}}, \frac{\mathrm{d} q_{\mathrm{p}}}{\mathrm{d} t}\right) & =2 a_{b}^{2} U_{0} \Delta_{U} R_{\mathrm{p}}\left(\frac{f_{1}\left(q_{\mathrm{p}}\right)-2 L_{\text {hor }}^{2}}{f_{1}^{2}\left(q_{\mathrm{p}}\right)}-\frac{f_{2}\left(q_{\mathrm{p}}\right)-2 L_{\text {hor }}^{2}}{f_{2}^{2}\left(q_{\mathrm{p}}\right)}\right) \frac{\mathrm{d} q_{\mathrm{p}}}{\mathrm{d} t} \\
f_{1}\left(q_{\mathrm{p}}\right) & =\left(q_{\mathrm{p}}-R_{\mathrm{p}}+L_{\text {ver }}\right)^{2}+L_{\text {hor }}^{2} \\
f_{2}\left(q_{\mathrm{p}}\right) & =\left(q_{\mathrm{p}}+R_{\mathrm{p}}+L_{\text {ver }}\right)^{2}+L_{\text {hor }}^{2} .
\end{aligned}
$$

Notice the movement of the sphere is not affected by the magnetic field. Finally, the system composed of the magnet, the coil and the moving sphere is modeled as a constant source of magneto-motive force $H_{\mathrm{p}}^{\text {mag }}=\frac{B_{\mathrm{p}}^{\text {mag }}}{U_{0}}$, connected to a gyrator modulated by the sphere position and velocity:

$$
\left(\begin{array}{c}
\frac{\mathrm{d} \phi_{\mathrm{p}}}{\mathrm{d} t_{\text {mag }}} \\
-\frac{\mathrm{d} \phi_{0}^{\text {mag }}}{\mathrm{d} t}
\end{array}\right)=\left(\begin{array}{cc}
0 & f_{\mathrm{p}} \\
-f_{\mathrm{p}} & 0
\end{array}\right)\left(\begin{array}{c}
h_{\text {coil }} \\
H_{\mathrm{p}}^{\text {mag }}
\end{array}\right) .
$$


Port-Hamiltonian modeling. The port-Hamiltonian modeling of the pickup connected to the $\mathrm{RC}$ circuit is made of two storage components (magnetic and electric capacitances with magnetic flux $\phi_{\text {coil }}$ and electric charge $q_{C}$ as respective states), one resistive element (electric resistance $R_{\mathrm{RC}}$ ) and two ports (constant magnetomotive force due to the magnet $H_{\mathrm{p}}^{\mathrm{mag}}$ and zero input current [39]), as depicted in figure 5. The nonlinearity of this system is only due to the modu270 lating coefficient $f_{p}$ in gain matrix $\mathbf{G}$. The resulting port-Hamiltonian system is given in table 7 (see also figure 5), with parameters in table 6 .

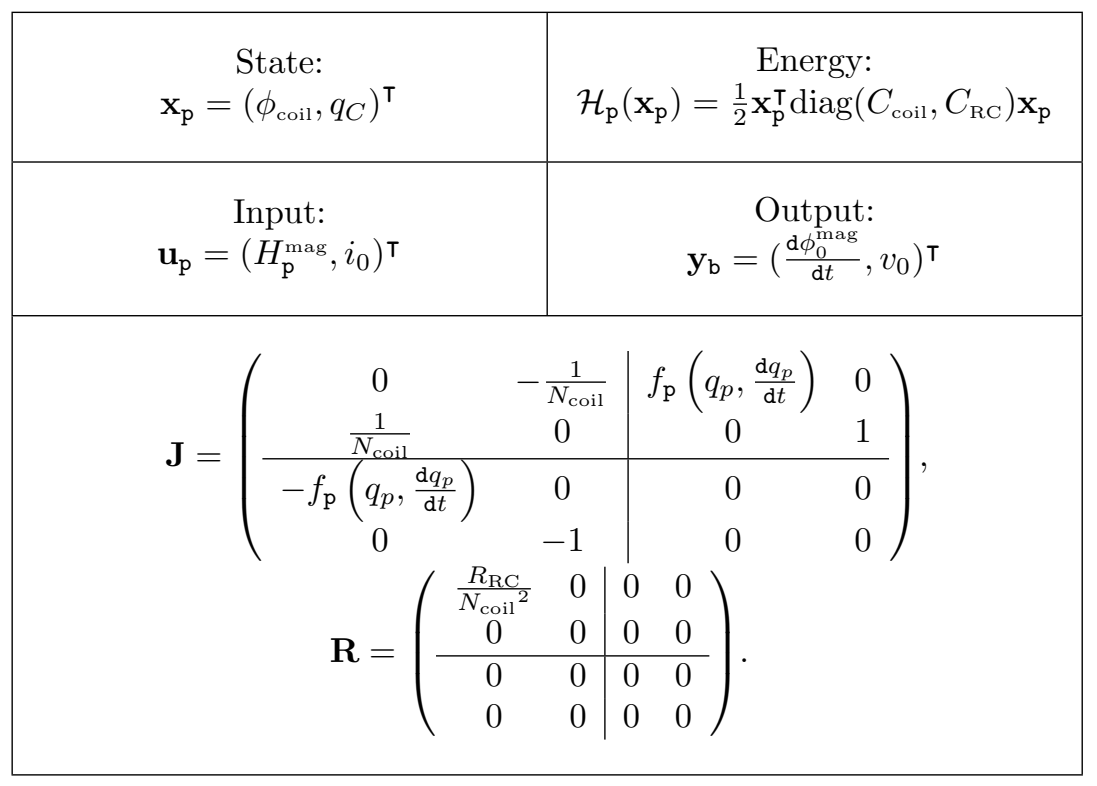

Table 7: Port-Hamiltonian formulation 56 for the pickup $\mathrm{p}$, with $\phi_{\text {coil }}$ the magnetic flux in the coil, $q_{C}$ the charge of the capacitor, $H_{\mathrm{p}}^{\mathrm{mag}}$ the magnetomotive force imposed by the magnet and $v_{0}$ the output voltage (see figure 5). The expression for $f_{\mathrm{p}}$ is given in equation 25), with $q_{p}$ the reconstruction of deflect measured at the beam free end $\left(z=L_{\mathrm{b}}\right)$. In this case, the invariant subspace 77 is $\mathbb{S}_{\mathrm{p}}=\{\mathbb{O}\} \subset \mathbb{R}^{2}$.

\section{Complete system}

In this section we derive the global port-Hamiltonian modeling of the system $(\mathrm{h}, \mathrm{b}, \mathrm{p})$ from the interconnection of the elementary $\mathrm{pH}$ systems derived in section 275 3. First we connect the mechanical components (h, b) and second the pickup which is not energetically but geometrically coupled to the former part. Notice the connection of two $\mathrm{pH}$ systems is again a $\mathrm{pH}$ system (see [44]). The total state is the concatenation of the subsystems states, and the total Hamiltonian is the sum of the subsystems Hamiltonians. A conservative interconnection is 280 then achieved by connecting each port of one system to exactly one port of the other. 
The connection of the hammer with the beam is as follows. The velocity of the contact point $v_{\mathrm{ext}}^{\text {cont }}$ that corresponds to the second input of the hammer (see table 3) is the reconstruction of the beam velocity $v_{\mathrm{b}}^{\text {ext }}$, that is, the output of the beam (see table 5): $v_{\mathrm{ext}}^{\text {cont }}=\left(\mathbb{0}, \Omega^{\top}\right) \nabla \mathcal{H}_{\mathrm{b}}\left(\mathrm{x}_{\mathrm{b}}\right)$. Correspondingly, the input force of the beam is the force experienced by the contact point of the hammer $f_{\mathrm{b}}^{\text {ext }}=-f_{\text {ext }}^{\text {cont }}$. This yields the following relations (see tables 3 and 5 ):

$$
\left\{\begin{array}{l}
{\left[\mathbf{u}_{\mathrm{h}}\right]_{2}=\left[\mathbf{y}_{\mathrm{b}}\right]_{1}=\left(\mathbb{O}, \Omega^{\top}\right) \nabla \mathcal{H}_{\mathrm{b}}\left(\mathbf{x}_{\mathrm{b}}\right)} \\
{\left[\mathbf{u}_{\mathrm{b}}\right]_{1}=-\left[\mathbf{y}_{\mathrm{h}}\right]_{2}=\left(r_{\mathrm{h}}\left(x_{\mathrm{h}, 2}\right), 1\right) \nabla \mathcal{H}_{\mathrm{h}}\left(\mathbf{x}_{\mathrm{h}}\right)-r_{\mathrm{h}}\left(x_{\mathrm{h}, 2}\right)\left(\mathbb{O}, \Omega^{\top}\right) \nabla \mathcal{H}_{\mathrm{b}}\left(\mathbf{x}_{\mathrm{b}}\right) .}
\end{array}\right.
$$

Notice this yields a passive interconnection in the sense that the power lost by the hammer is exactly that received by the beam $\left[\mathbf{u}_{\mathrm{b}}\right]_{1}\left[\mathbf{y}_{\mathbf{b}}\right]_{1}=-\left[\mathbf{u}_{\mathrm{h}}\right]_{1}\left[\mathbf{y}_{\mathrm{h}}\right]_{1}$. The connection (29) is then replaced in the diagonal concatenation of structure 285 matrices $\mathbf{J}$ and $\mathbf{R}$ from tables 3 and $\mathbf{5}$.

As already stated, the mechanical part is not energetically coupled to the electromagnetic part, and the complete modeling is obtained by concatenating the interconnection $(\mathrm{h}, \mathrm{b})$ with the pickup $\mathrm{p}$. The arguments of the modulation

290 coefficient $f_{\mathrm{p}}\left(q_{p}, \frac{\mathrm{d} q_{p}}{\mathrm{~d} t}\right)$ (see table 7 ) is obtained from the reconstruction of the movement of the beam free end with $q_{p}=q\left(L_{\mathrm{b}}\right)$. Finally, the port-Hamiltonian modeling of the system depicted in figure 2 is given in table 8 .

\section{Guaranteed passive numerical method}

Numerous numerical methods have been developed to simulate dynamical systems governed by $\frac{\mathrm{dx}(t)}{\mathrm{d} t}=\mathbf{f}(\mathbf{x}(t), t)$, to approximate the solution $\mathbf{x}\left(t_{k}\right)$, on e.g. a regular grid defined by $t_{k}=k T, k \in \mathbb{Z}$. Many of these methods are based on an approximation of $\frac{\mathrm{d}}{\mathrm{d} t}$ and exploit the vector field $\mathbf{f}$ at specific instants $t \in\left[t_{k}, t_{k+1}\right]$, but do not preserve passivity. This section introduces a method that preserves the power balance (1) in the discrete time-domain, so that a numerical power balance holds:

$$
\frac{\mathcal{E}\left(t_{k+1}\right)-\mathcal{E}\left(t_{k}\right)}{T}=-\mathcal{Q}\left(t_{k}\right)+\mathcal{P}_{\text {ext }}\left(t_{k}\right) .
$$

The approach presented below is known as the discrete gradient method introduced for classical Hamiltonian systems in [45] (see also [46, 47] and [48, §4.3]).

\subsection{Numerical scheme}

Standard first order approximation of the differentials $\mathrm{dx}(t, \mathrm{~d} t)=\frac{\mathrm{d} \mathbf{x}}{\mathrm{d} t}(t) \cdot \mathrm{d} t$ and $\mathrm{d} \mathcal{H}(\mathbf{x}, \mathrm{d} \mathbf{x})=\nabla \mathcal{H}(\mathbf{x})^{\boldsymbol{\top}} \cdot \mathrm{d} \mathbf{x}$ are given by $\delta \mathbf{x}\left(t_{k}\right)=\mathbf{x}\left(t_{k+1}\right)-\mathbf{x}\left(t_{k}\right)$, and $\delta \mathcal{H}(\mathbf{x}, \delta \mathbf{x})=$ $\mathcal{H}(\mathbf{x}+\delta \mathbf{x})-\mathcal{H}(\mathbf{x})=\nabla_{d} \mathcal{H}(\mathbf{x}, \delta \mathbf{x})^{\top} \cdot \delta \mathbf{x}$, respectively. For mono-variate storage components with $\mathcal{H}\left(\mathbf{x}\left(t_{k}\right)\right)=\sum_{n=1}^{N_{\mathcal{E}}} \mathcal{H}_{n}\left(x_{n}\left(t_{k}\right)\right)$ (this is the case for the system 


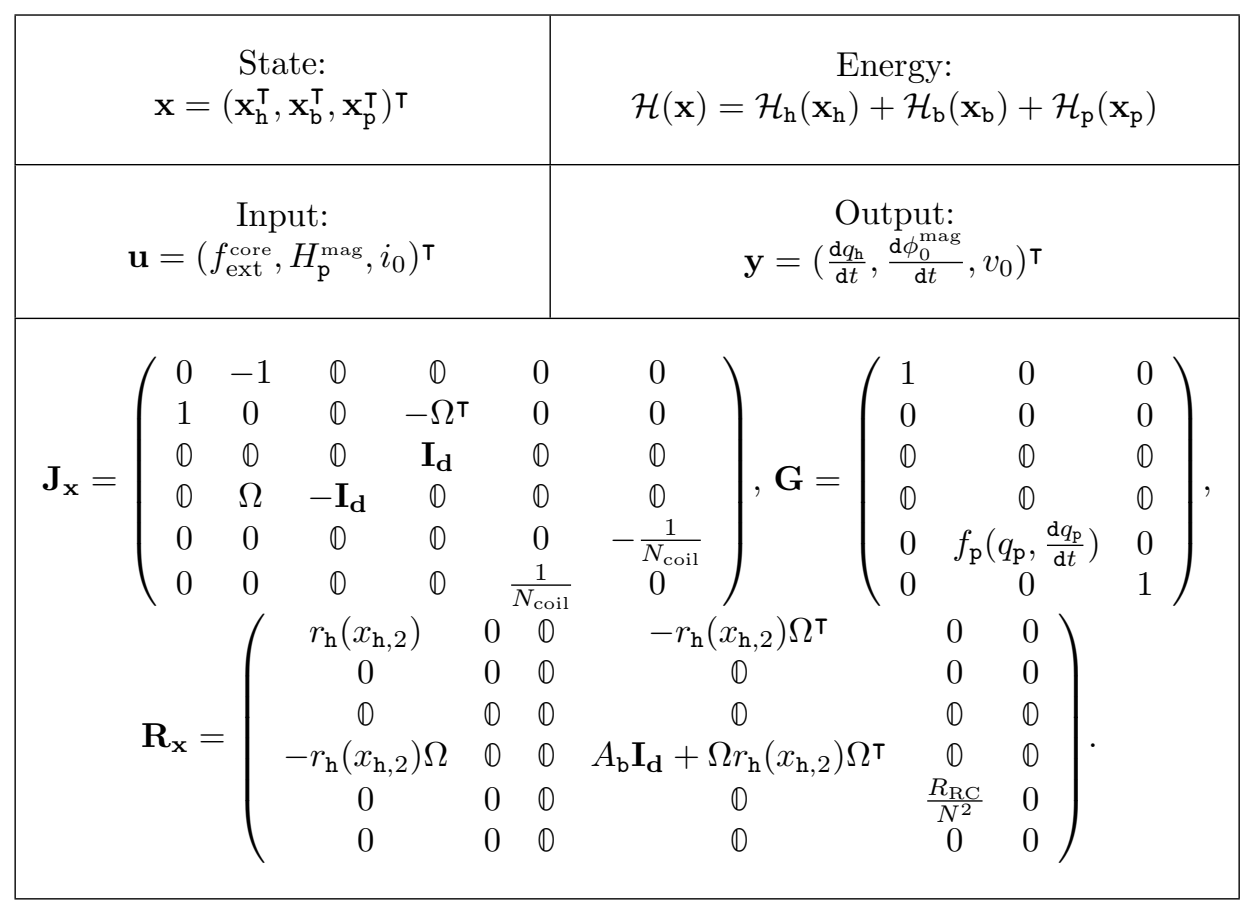

Table 8: Port-Hamiltonian formulation $\sqrt{5} 6$ for the complete system (h, b, p, RC) (see tables 3 , 5 and 7 for definitions), where unspecified matrices are zeros. Here, the invariant subspace (7) is the union of the invariant sets of the components so that $\mathbb{S}_{\text {rhodes }}=\mathbb{S}_{\mathrm{h}} \times \mathbb{S}_{\mathrm{b}} \times \mathbb{S}_{\mathrm{p}}=$ $\left\{\mathbf{x} \in \mathbb{R}^{2+2+2 M}\right.$ s.t. $\left.[\mathbf{x}]_{2} \leq 0,[\mathbf{x}]_{i}=0, i \neq 2\right\}$, that is, the hammer goes to minus infinity with constant velocity after it has rebounded on the beam, and the latter with pickup circuit return to equilibrium.

described in table 8), a discrete gradient is introduced and defined by

$$
\left[\nabla_{d} \mathcal{H}\left(\mathbf{x}\left(t_{k}\right), \delta \mathbf{x}\left(t_{k}\right)\right)\right]_{n}= \begin{cases}\frac{\mathcal{H}_{n}\left(x_{n}\left(t_{k}\right)+\delta x_{n}\left(t_{k}\right)\right)-\mathcal{H}_{n}\left(x_{n}\left(t_{k}\right)\right)}{\delta x_{n}\left(t_{k}\right)} & \text { if } \delta x_{n}\left(t_{k}\right) \neq 0 \\ \mathcal{H}_{n}^{\prime}\left(x_{n}\left(t_{k}\right)\right) & \text { otherwise }\end{cases}
$$

so that a discrete chain rule is recovered

$$
\frac{\delta \mathcal{E}\left(t_{k}\right)}{T}=\nabla_{d} \mathcal{H}\left(\mathbf{x}\left(t_{k}\right), \delta \mathbf{x}\left(t_{k}\right)\right)^{\top} \frac{\delta \mathbf{x}\left(t_{k}\right)}{T} .
$$

Then, applying the following substitution in (5 6)

$$
\begin{aligned}
\frac{\mathrm{dx}\left(t_{k}\right)}{\mathrm{d} t} & \rightarrow \frac{\delta \mathbf{x}\left(t_{k}\right)}{T} \\
\nabla \mathcal{H}\left(\mathbf{x}\left(t_{k}\right)\right) & \rightarrow \nabla_{d} \mathcal{H}\left(\mathbf{x}\left(t_{k}\right), \delta \mathbf{x}\left(t_{k}\right)\right) \equiv \nabla_{d} \mathcal{H}_{k},
\end{aligned}
$$


leads to

$$
\begin{aligned}
\left(\begin{array}{c}
\nabla_{d} \mathcal{H}_{k} \\
\mathbf{u}\left(t_{k}\right)
\end{array}\right)^{\top}\left(\begin{array}{c}
\frac{\delta \mathbf{x}\left(t_{k}\right)}{\delta t} \\
-\mathbf{y}\left(t_{k}\right)
\end{array}\right) & =\underbrace{\nabla_{d} \mathcal{H}_{k}^{\top} \frac{\delta \mathbf{x}\left(t_{k}\right)}{\delta t}}_{\nabla_{d}\left(t_{k}\right)}-\underbrace{\mathbf{u}\left(t_{k}\right)^{\top} \mathbf{y}\left(t_{k}\right)}_{\mathcal{P}_{\text {ext }}\left(t_{k}\right)} \\
& =-\underbrace{\left(\begin{array}{c}
\nabla_{d} \mathcal{H}_{k} \\
\mathbf{u}\left(t_{k}\right)
\end{array}\right)^{\top} \mathbf{R}\left(\mathbf{x}\left(t_{k}\right)\right)\left(\begin{array}{c}
\nabla_{d} \mathcal{H}_{k} \\
\mathbf{u}\left(t_{k}\right)
\end{array}\right)}_{\mathcal{Q}\left(t_{k}\right)}
\end{aligned}
$$

(same as proof of property 2.1). For a conservative system $(\mathbf{R}=\mathbb{0}, \mathbf{G}=\mathbb{0}$, $\mathbf{J}_{\mathbf{y}}=\mathbb{0}$ ), this method yields $\delta \mathcal{E}\left(t_{k}\right)=0$ so that $\mathcal{E}\left(t_{k+1}\right)=\mathcal{E}\left(t_{k}\right)$ (the energy is conserved). For a passive system $\left(\mathbf{R} \neq \mathbb{0}, \mathbf{G} \neq \mathbb{0}, \mathbf{J}_{\mathbf{y}} \neq \mathbb{0}\right)$, this method restores a discrete version of property 2.1 so that observations of remark 1 holds. Notice the invariant set $\mathbb{S}_{\text {rhodes }}$ is the same as in the continuous time domain (see caption of table 8).

The associated update is

$$
\begin{aligned}
\mathbf{x}\left(t_{k+1}\right) & =\mathbf{x}\left(t_{k}\right)+\delta \mathbf{x}\left(t_{k}\right) \\
\delta \mathbf{x}\left(t_{k}\right) & =T\left(\mathbf{J}_{\mathbf{x}}-\mathbf{R}_{\mathbf{x}}\right) \nabla_{d} \mathcal{H}\left(\mathbf{x}\left(t_{k}\right), \delta \mathbf{x}\left(t_{k}\right)\right)+T\left(\mathbf{G}-\mathbf{R}_{\mathbf{x y y}}\right) \mathbf{u}\left(t_{k}\right) \\
\mathbf{y}\left(t_{k}\right) & =\left(\mathbf{G}+\mathbf{R}_{\mathbf{x y}}\right)^{\top} \nabla_{d} \mathcal{H}\left(\mathbf{x}\left(t_{k}\right), \delta \mathbf{x}\left(t_{k}\right)\right)-\left(\mathbf{J}_{\mathbf{y}}-\mathbf{R}_{\mathbf{y}}\right) \mathbf{u}\left(t_{k}\right)
\end{aligned}
$$

where state-dependent matrices are evaluated at $\mathbf{x}\left(t_{k}\right)$, and the implicit relation on $\delta \mathbf{x}\left(t_{k}\right)$ is solved at each time step by a fixed number of Newton-Raphson iterations. Notice the Rhodes model in table 8 includes a single nonlinear storage component (spring effect associated with the hammer felt). In this case, the Newton-Raphson algorithm only requires the inversion of a scalar function, that allows real-time applications.

\subsection{Convergence analysis}

Although the discrete power balance ensures the asymptotic stability of the numerical solution, it does not provide information on the convergence of the sequence $\mathbf{x}\left(t_{k}\right)$ to the continuous solution. From Lax's theorem, convergence holds provided (i) the method is consistent with the system (5 6) and (ii) the total error can be bounded (stability of the method). From [49, (3), §4.2], the method that yields the slope $\boldsymbol{\sigma}\left(t_{k}\right)=\frac{\delta \mathbf{x}_{k}}{T}$ is consistent if and only if

$$
\lim _{T \rightarrow 0} \boldsymbol{\sigma}\left(t_{k}, T\right)=\mathbf{f}\left(\mathbf{x}\left(t_{k}\right), t_{k}\right)
$$

Assuming that $\mathcal{H}$ satisfies a Lipschitz condition (this is the case for the system in table 8), the discrete gradient is consistent with the continuous gradient:

$$
\lim _{\|\delta \mathbf{x}\|_{2} \rightarrow 0} \nabla_{d} \mathcal{H}(\mathbf{x}, \delta \mathbf{x})=\nabla \mathcal{H}(\mathbf{x})
$$

Now, provided the structure matrices $\mathbf{J}(\mathbf{x})$ and $\mathbf{R}(\mathbf{x})$ are bounded (this is the case for the system in table 8$)$, the slope $\boldsymbol{\sigma}\left(t_{k}\right)$ is bounded so that $\lim _{T \rightarrow 0} \delta \mathbf{x}\left(t_{k}\right)=$ 
0 . This proves the numerical method is consistent up to order 1. Additionally, the method (33) proves stable (in the sense that the total error due to the accumulation of round-off and truncation errors is bounded) from the Lipschitz property of the Hamiltonian $\mathcal{H}$ and the structure matrices (see [49, theorem 4.1]). This proves the convergence of the discrete gradient method for port-Hamiltonian systems (5 6).

Remark 2 (Second order). Order 2 is achieved for constant matrices $\mathbf{J}$ and $\mathbf{R}$, and can be recovered for non constant structure with an additional computation step of Runge-Kutta type (see [15] for details).

Remark 3 (Coincidence with the midpoint rule). For port-Hamiltonian systems composed of a collection of linear energy storing components with quadratic Hamiltonian $\mathcal{H}_{n}\left(x_{n}\right)=\frac{x_{n}^{2}}{2 C_{n}}$, we define $\mathbf{Q}=\operatorname{diag}\left(C_{1} \cdots C_{N_{\mathcal{E}}}\right)^{-1}$ so that the discrete gradient (31) reads

$$
\nabla_{d} \mathcal{H}(\mathbf{x}, \mathbf{x}+\delta \mathbf{x})=\mathbf{Q}\left(\mathbf{x}(k)+\frac{\delta \mathbf{x}(k)}{2}\right) .
$$

For a constant structure, this restores the midpoint rule $\frac{\delta \mathbf{x}_{k}}{T}=\mathbf{f}\left(\frac{\mathbf{x}_{k}+\mathbf{x}_{k+1}}{2}\right)$ that coincides in this case with the trapezoidal rule $\frac{\delta \mathbf{x}_{k}}{T}=\frac{\mathbf{f}\left(\mathbf{x}_{k}\right)+\mathbf{f}\left(\mathbf{x}_{k+1}\right)}{2}$. However, for nonlinear cases, (31) leads to a class of numerical schemes depending on the nonlinearity, still preserving passivity. In these cases, the discrete gradient approach does not coincide anymore with the midpoint rule, which also does not coincides anymore with the trapezoidal rule.

\section{Results}

In this section, the numerical scheme (33) is applied on the modeling presented in section 4 . The sample rate is $T^{-1}=48 \mathrm{kHz}$. Physical parameters are given in tables 2 , 4 and 6 . They are chosen to correspond to the musical note A4 $(440 \mathrm{~Hz})$. The basis of eigenmodes is truncated so that the highest eigenfrequency is below the Nyquist frequency, which yields $M=4$ modes for note A4 at $48 \mathrm{kHz}$. The corresponding eigenfrequencies are $\nu_{1}=440 \mathrm{~Hz}, \nu_{2} \simeq 2757.49 \mathrm{~Hz}$, ${ }_{335} \quad \nu_{3} \simeq 7721.07 \mathrm{~Hz}$ and $\nu_{4} \simeq 15130.22 \mathrm{~Hz}$ (see $\S 3.2$ and appendix AppendixA.1). Audio example are available her ${ }^{2}$. The input force $f_{\text {ext }}^{\text {core }}$ is comprised between 0 and $10^{3} \mathrm{~N}$, during $1 \mathrm{~ms}$. A simple exhaust mechanism is included (not shown in tables 3 and 8 with $f_{\text {ext }}^{\text {core }}=0$ if $q_{h}>-0.5 \mathrm{~cm}$.

\subsection{Mechanical energy}

340 The dynamics of the hammer is shown in figure 7 for $f_{\text {ext }}^{\text {core }}=500 \mathrm{~N}$. We see it accelerates between $1 \mathrm{~ms}$ and $2 \mathrm{~ms}$ and impacts the beam at $t_{i} \simeq 2.5 \mathrm{~ms}$. During the impact, a part of the energy transferred from the hammer to the beam is dissipated (see figure 8a). The energy in the beam is shown in figure $8 \mathrm{~b}$ We see that a numerical energy balance is fulfilled.

2 http://recherche.ircam.fr/anasyn/falaize/applis/rhodes/index.html 


\section{Dynamics of the hammer}
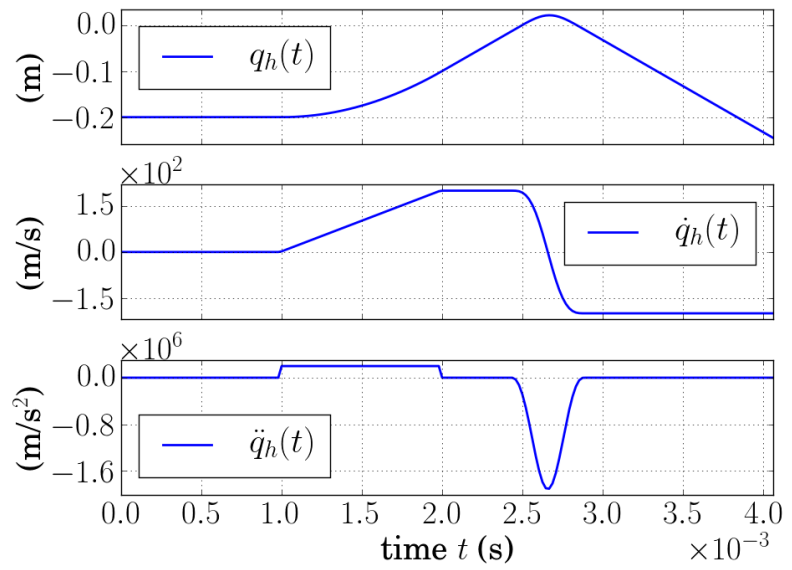

Figure 7: Position, velocity and acceleration of the hammer.

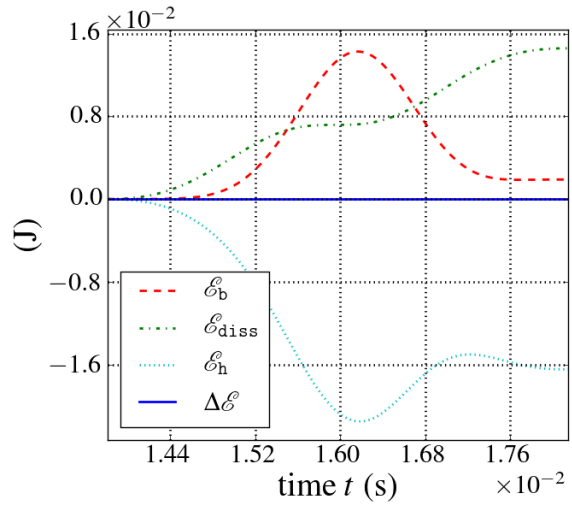

(a) Mechanical energy at impact.

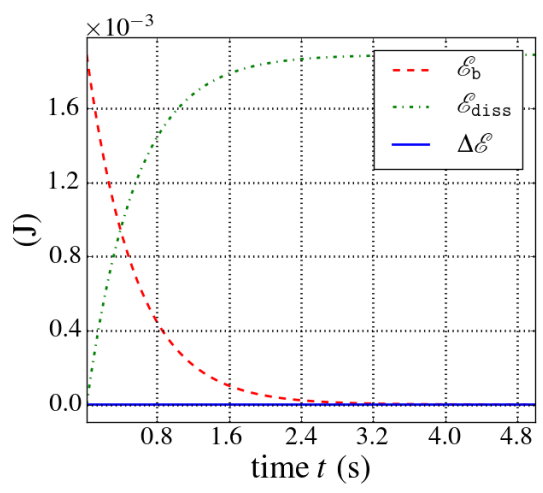

(b) Mechanical energy after impact.

Figure 8: Evolution of the mechanical energy. $\mathcal{E}_{\mathrm{b}}$ is the energy of the beam, $\mathcal{E}_{\text {diss }}$ is the dissipated energy $\int \mathcal{Q} \mathrm{d} t, \mathcal{E}_{\mathrm{h}}$ is the increment on the energy of the hammer $\mathcal{H}_{\mathrm{h}}\left(\mathbf{x}_{\mathrm{h}}(t)\right)-\mathcal{H}_{\mathrm{h}}\left(\mathbf{x}_{\mathrm{h}}\left(t_{0}\right)\right)$ with $t_{0}$ the impact moment and $\Delta \mathcal{E}$ is the energy balance $\mathcal{E}_{\mathrm{b}}+\mathcal{E}_{\mathrm{h}}+\mathcal{E}_{\mathrm{diss}}$.

\subsection{Electromagnetic energy}

The source of constant magnetomotive force is modulated according to section 3.3 Note such a source can be locally a sink of power, as seen in figure 9 where the power passes slightly under 0W. Again, the numerical error on the power balance is close to the machine epsilon. 


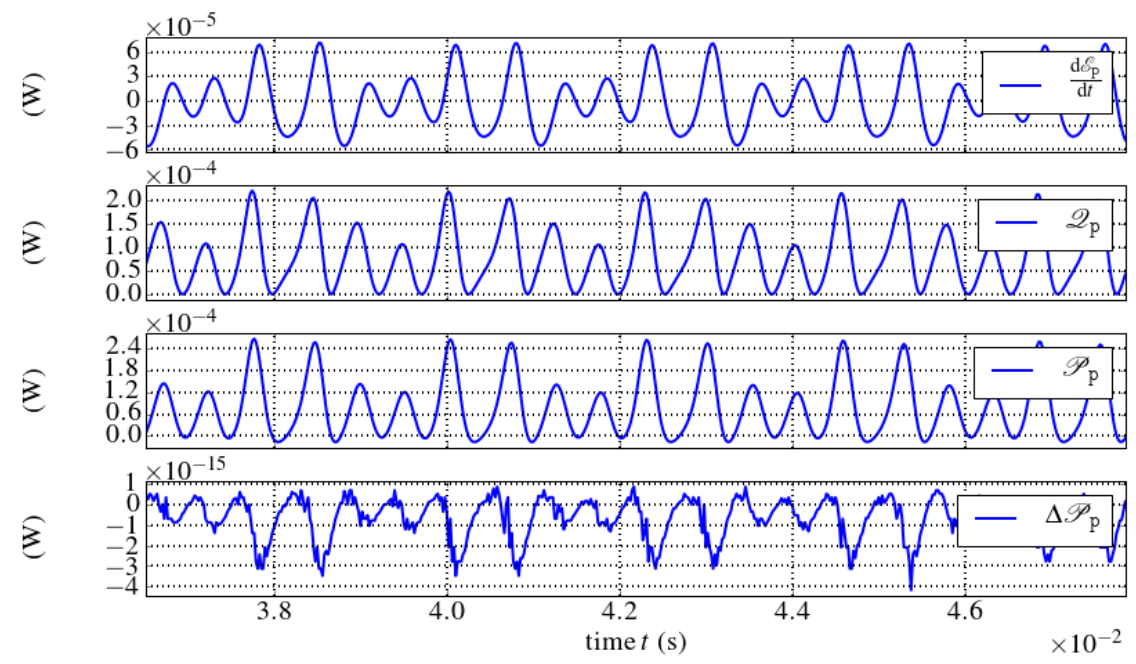

Figure 9: (Simulation) Power balance for the electro-magnetic part (pickup and RC ana$\log$ circuit) with energy variation $\frac{\mathrm{d} \mathcal{E}_{\mathrm{p}}}{\mathrm{d} t}=\nabla \mathcal{H}_{\mathrm{p}}^{\mathrm{\top}} \frac{\mathrm{dx} \mathrm{p}}{\mathrm{d} t}$, dissipated power $\mathcal{Q}_{\mathrm{p}}=\frac{R_{\mathrm{RC}}}{N_{\text {coil }}{ }^{2}}\left[\nabla \mathcal{H}_{\mathrm{p}}\right]_{1}^{2}$, source power $\mathcal{P}_{\mathrm{p}}=\mathbf{u}_{\mathrm{p}}^{\top} \mathbf{y}_{\mathrm{p}}$ (see table 7), and deviation on the power balance $\Delta \mathcal{P}_{\mathrm{p}}=$ $\left(\frac{\mathrm{d} \mathcal{E}_{\mathrm{p}}}{\mathrm{d} t}+\mathcal{Q}_{\mathrm{p}}-\mathcal{P}_{\mathrm{p}}\right) /\left\langle\mathcal{P}_{\mathrm{p}}\right\rangle$, relative to the the mean source power $\left\langle\mathcal{P}_{\mathrm{p}}\right\rangle \simeq 10^{-4} \mathrm{~W}$ for the five periods shown in the figure.

\subsection{Output signal}

The output voltage $v_{0}$ is proportional to the beam free-end velocity multiplied by a nonlinear function of the position $q_{\mathrm{p}}=q\left(z=L_{\mathrm{b}}\right)$ given by (25). Since the beam modeling is linear (see section 3.2), this transduction mechanism is the only one that is responsible for the characteristic Rhodes piano tones, as noticed in 19, 20. Figure 10 displays the displacement and the velocity of the beam free-end as well as the output voltage $v_{0}$ (see figure 5 and tables 7 and 8 ). These signals have been computed for $f_{\text {ext }}^{\text {core }}=500 \mathrm{~N}$ (note A4). We can notice in figure $10 \mathrm{~b}$ that higher modes of the resonator are not extensively excited, and that the distinctive evolution of the tone is due to the pickup only.

\subsection{Comparison with measurements}

The physical modeling approach to sound synthesis provides an easy tuning of the model parameters to simulate a given device. This has been done based on measurements provided by UVl $\mathrm{l}^{3}$, a company specialized in building samplebased real-time synthesizers of musical instruments. A rough comparison of spectrograms for increasing hammer force (from $100 \mathrm{~N}$ to $1000 \mathrm{~N}$ in the $\mathrm{pH}$ model) is shown in figure11. A detailed comparison of the normalized waveforms in the cases force 1 , force 4 and force 7 is given in figure 12 .

\footnotetext{
${ }^{3}$ http://www.uvi.net/
} 


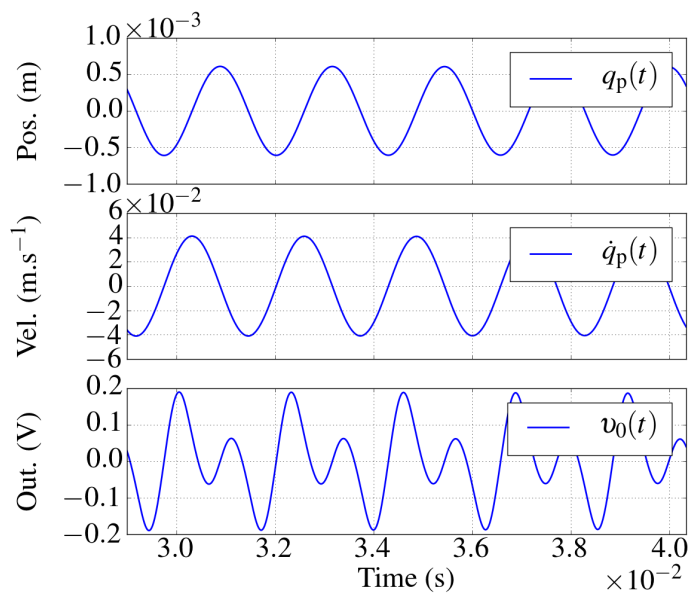

(a) Reconstruction of beam displacement and velocity at the beam free end $z=L_{\mathrm{b}}$, and output voltage $v_{0}$.

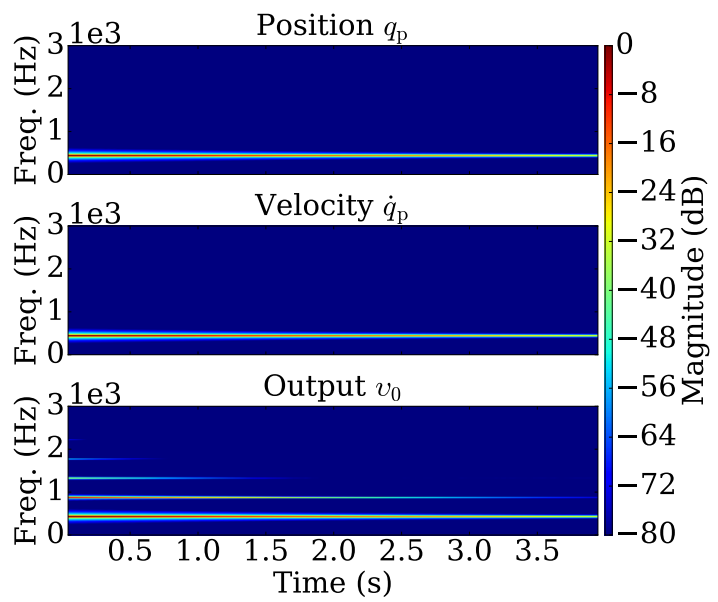

(b) Normalized spectra obtained from fast Fourier transform on $2^{11}$ samples with overlap ratio $50 \%$.

Figure 10: (Simulation) Displacement $q_{\mathrm{p}}=q\left(z=L_{\mathrm{b}}\right)$ and velocity $\frac{\mathrm{d} q_{\mathrm{p}}}{\mathrm{d} t}$ of the free end of the beam, and corresponding output voltage $v_{0}$ that results from the pickup non-linearity 25 .

\subsection{Playing with the model}

According to the Fender Rhodes manual, the position of the pickup with respect to the beam's axis has a critical effect on the resulting timbre and transients of the output voltage. This can be easily verified in our simulations. Results for the horizontal $L_{\text {hor }}$ and vertical alignment $L_{\text {ver }}$ prove in good accordance with predicted behaviors as shown in figures 13.

\section{Conclusions}

In this paper, a nonlinear finite-dimensional model of a simplified electromechanical piano has been developed, based on a set of elementary components (hammer, beam and pickup), in the framework of port Hamiltonian systems. This formalism splits the system into conservative, dissipative and source parts. A numerical method has been derived, which preserves this decomposition and the power balance in the discrete time domain. The analysis of numerical results proves the relevancy of the method: first, the analysis of the power exchanges and of the total energy shows that passivity is fulfilled; second, the numerical scheme proves compatible with real-time purposes; third, results are consistent with measurements on a real device.

385

Several model sophistications and refinements could be considered in perspectives of this work, some of which are listed thereafter in order of importance with respect to the sound realism. First of all is the modeling of both prongs for the resonator (elements $7-13$ in figure 1) with their energy transfer. This 

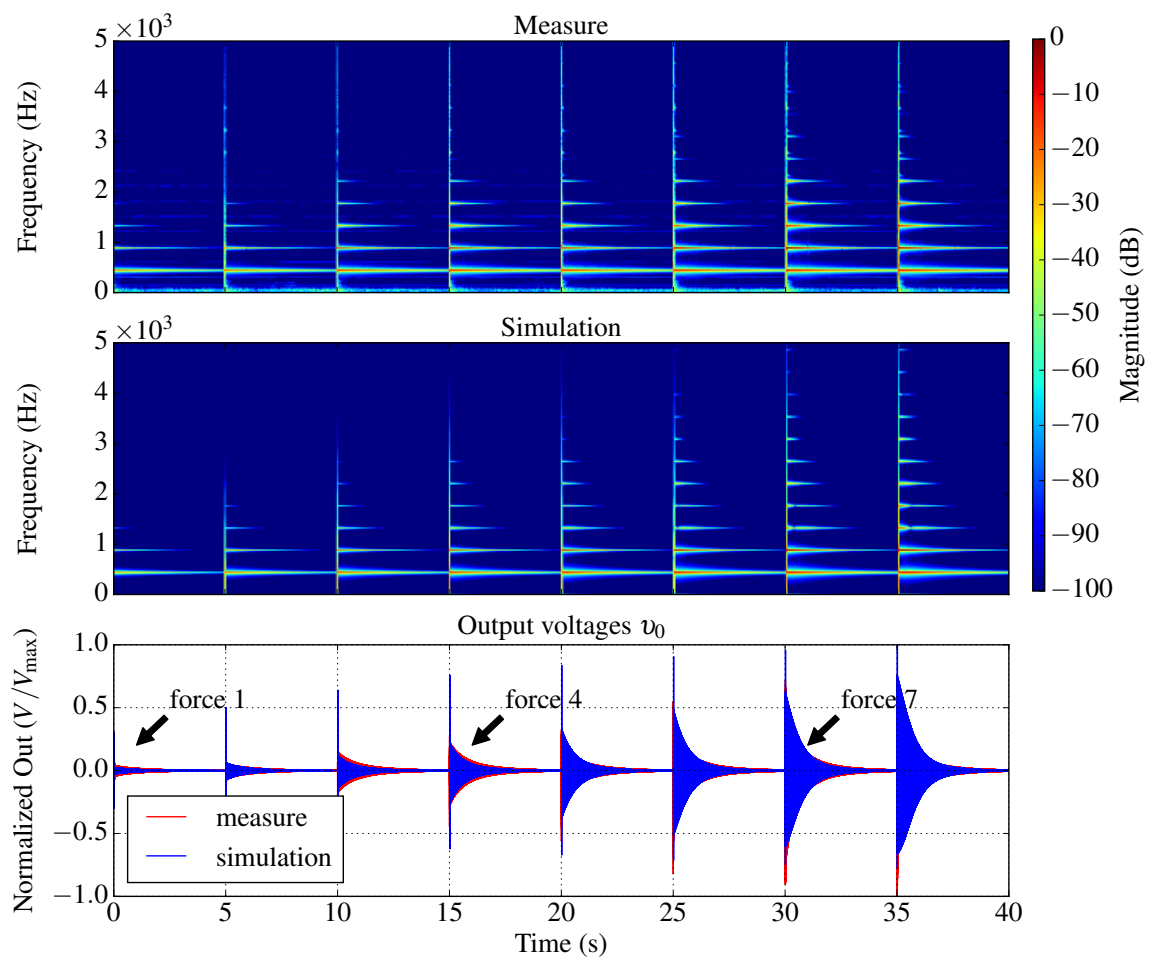

Figure 11: Spectral densities for the reference (upper) and simulation (middle), and normalized output voltages (lower). See figure 12 for a detailed comparison of waveforms in the cases force 1 , force 4 and force 7 . The dynamics for the spectrograms is $100 \mathrm{~dB}$, which is perceptively relevant for audio applications. We see the recorder tones (upper) exhibits additional spectral content, mostly on the band $[1-2] \mathrm{kHz}$. This could come from the double polarization of the beam movement or from the coupling with the tone bar, that are not taken into account in the proposed model. The low frequency content is associated with external noise induced in the recorded signals. Finally, we see the model could benefit from an eigenmode dependent damping coefficient $A_{\mathrm{b}} \equiv\left[A_{\mathrm{b}, m}\right]_{1 \leq m \leq M}$ to fit with the decay of partials in the recorded tones.

should directly impact the transient and damping of output signal, as pointed out in [19]. Another critical element for playing with the instrument is the damper module (elements $16-18$ and $22-25$ in figure 1 ), which permits to control the duration of the vibration. The modeling of the hammer felt can be reused to address this issue. A less critical refinement is concerned with the movement of the tine that is not perfectly planar, thus inducing polarization effects that can appear in the output signal. This leads to consider a three-dimensional model for the tine vibration, and to adapt the pickup model accordingly. Additionally, the shear stress due to the deflect of the tine should be included; it does not seem to be necessary to describe torsion nor compression waves, due to (i) the 400 filiform geometry and (ii) the axial excitation mechanism. The hammer model could be complemented by modeling the key action mechanism (see 50] and 

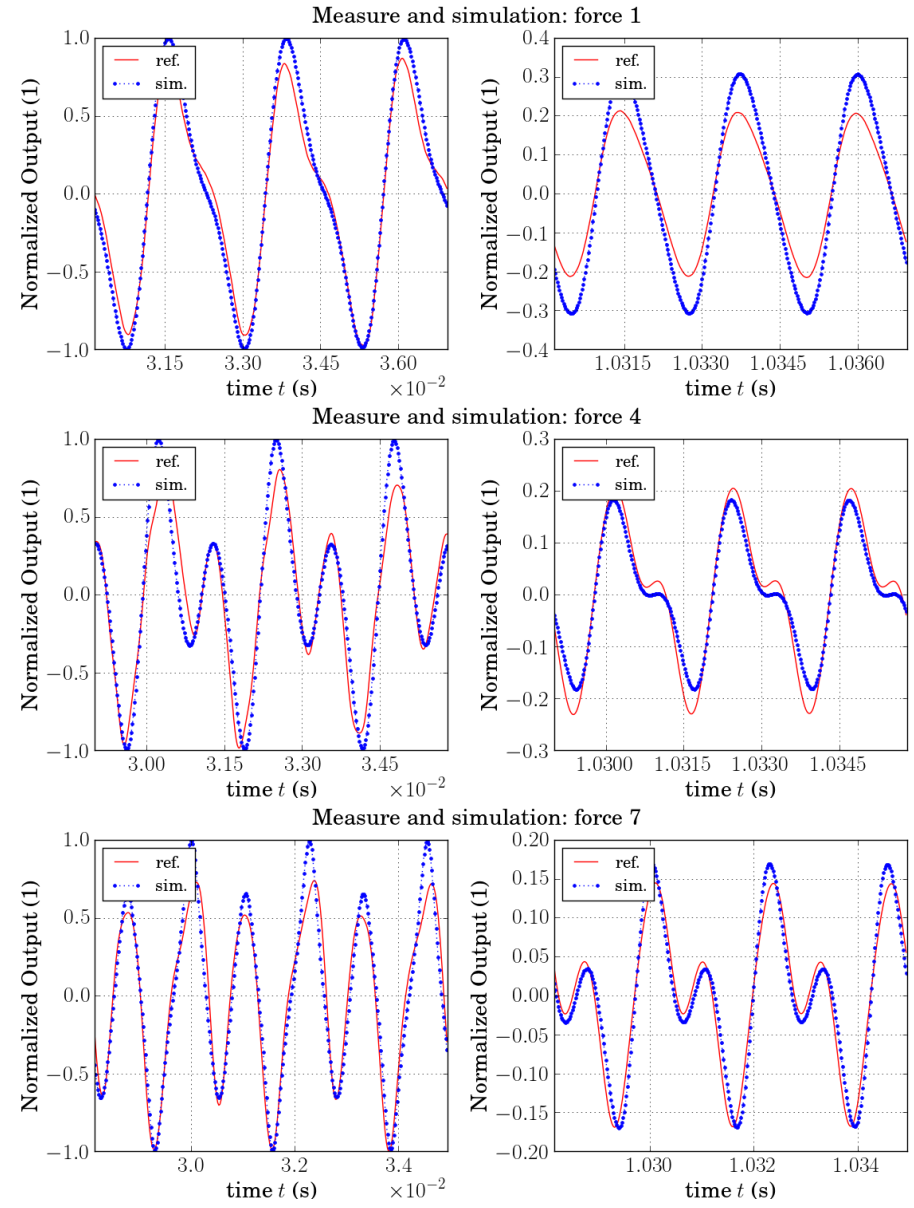

Figure 12: Comparison with reference signals (see figure 11 for correspondence of forces).

references therein), and the hammer felt model could be refined so as to exhibit a realistic asymptotic behavior (that is, the felt can not be infinitely crushed). Least of all, the mechano-electric transducer could be refined to include the energetic exchange due to the coupling between the beam and the magnetic field, by considering the Maxwell force. This should not alter the output signal except for increasing the damping; this point is to consider only in view of a comprehensive multi-physical model.

A second perspective is to look for a method to extract the physical parameters from the measurements, in order to increase the sound realism. A third perpective is to examine second order explicit numerical schemes (see e.g. [15]) to improve accuracy and reduce the computational cost. Finally, a playable instrument plugin could be developed based on $\mathrm{C}++$ implementation. 
Effect of pickup distance $L_{\mathrm{hor}}$
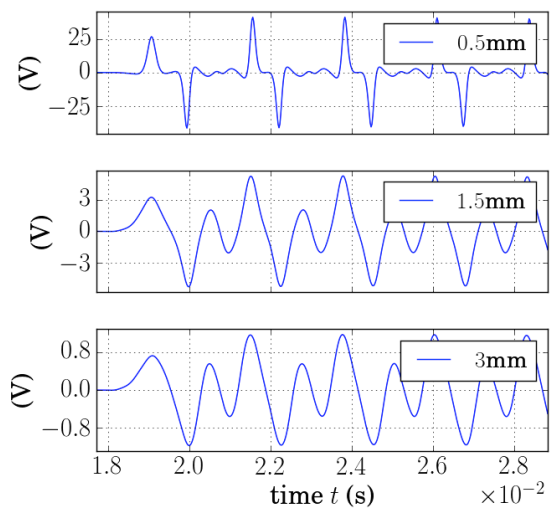

(a) With $L_{\text {ver }}=0.5 \mathrm{~mm}$ and $F_{h}=500 \mathrm{~N}$.

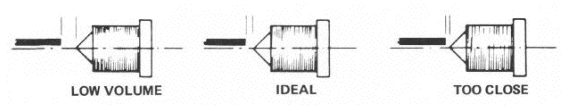

(c) Guide for tuning the distance $L_{\text {hor }}$.
Effect of vertical decay $L_{\text {ver }}$
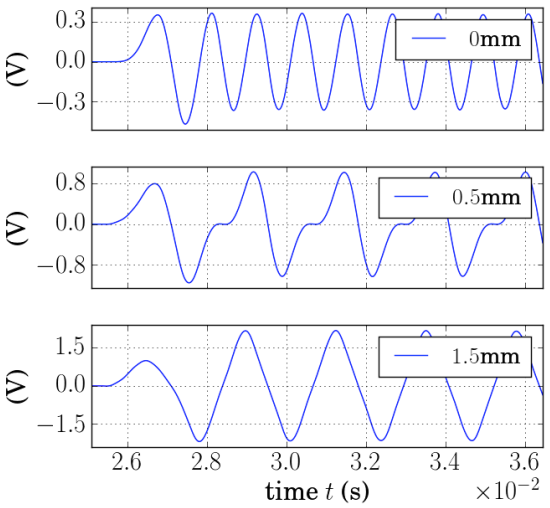

(b) With $L_{\text {hor }}=1 \mathrm{~mm}$ and $F_{h}=250 \mathrm{~N}$.

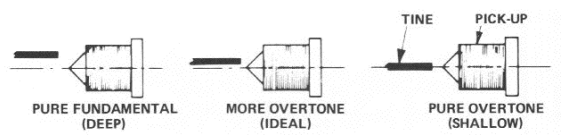

(d) Guide for tuning the distance $L_{\text {ver }}$

Figure 13: Effect of vertical alignment $L_{\mathrm{ver}}$ and horizontal distance $L_{\mathrm{hor}}$ on the output signal $v_{0}$.

\section{Acknowledgments}

The authors acknowledge the members of the french National Research Agency project HaMecMoPSys for support in port-Hamiltonian theory, Cyril Touzé for lectures on modal decomposition and UVI company (especially Damien Vallet) for providing high quality measurement of Rhodes tones. Last but not least, the authors acknowledge David Roze for careful proofreading.

\section{AppendixA. Modal decomposition}

\section{AppendixA.1. Orthonormal basis}

The spatial eigenvectors $\psi_{m}(z)$ which satisfy the boundary conditions of the cantilever beam (i-iv), $\partial_{z}^{4} \psi(z)=\kappa^{4} \psi(z)$ and $\left\langle\psi_{m}, \psi_{p}\right\rangle=\delta_{m, p}$ (Kronecker's symbol) for all $(m, p) \in \mathbb{N}_{*}^{2}$, where the scalar product on $L^{2}\left(0, L_{\mathrm{b}}\right)$ is defined by $\langle f, g\rangle=\int_{0}^{L_{\mathrm{b}}} f(z) g(z) \mathrm{d} z$ are

$$
\begin{aligned}
& \psi_{m}(z)=\gamma_{m} \widehat{\psi}_{m}(z) \\
& \widehat{\psi}_{m}(z)=\theta_{m}\left(\sin \kappa_{m} z-\sinh \kappa_{m} z\right)+\cos \kappa_{m} z-\cosh \kappa_{m} z
\end{aligned}
$$


for the normalizing coefficient

$$
\begin{aligned}
\gamma_{m}= & \left(\frac{\kappa_{m} L_{\mathrm{b}}\left(\cos 2 \kappa_{m} L_{\mathrm{b}}+\cosh 2 \kappa_{m} L_{\mathrm{b}}-2\right)}{2 \kappa_{m}\left(\cos \kappa_{m} L_{\mathrm{b}}+\cosh \kappa_{m} L_{\mathrm{b}}\right)^{2}}\right. \\
& \left.\frac{\cosh \kappa_{m} L_{\mathrm{b}}\left(2 \sin \kappa_{m} L_{\mathrm{b}}+\cosh \kappa_{m} L_{\mathrm{b}} \sin 2 \kappa_{m} L_{\mathrm{b}}\right)}{2 \kappa_{m}\left(\cos \kappa_{m} L_{\mathrm{b}}+\cosh \kappa_{m} L_{\mathrm{b}}\right)^{2}}\right)^{\frac{1}{2}}
\end{aligned}
$$

with $\cos \left(\kappa_{m} L_{\mathrm{b}}\right) \cosh \kappa_{m} L_{\mathrm{b}}+1=0$ and $\theta_{m}=\frac{\sin \left(\kappa_{m} L_{\mathrm{b}}\right)-\sinh \left(\kappa_{m} L_{\mathrm{b}}\right)}{\cos \left(\kappa_{m} L_{\mathrm{b}}\right)+\cosh \left(\kappa_{m} L_{\mathrm{b}}\right)}$.

\section{AppendixA.2. Projection}

The projection of a point force $f_{\mathrm{b}}^{\text {ext }}$ located at $z_{\mathrm{h}}$ and distributed according to $\sigma_{\mathrm{h}}(z)=\mathbb{1}\left(z-z_{\mathrm{h}}\right)_{\left[-D_{\mathrm{h}} / 2,+D_{\mathrm{h}} / 2\right]}$ on the truncated basis $\mathrm{B}=\left\{\psi_{m}\right\}_{1 \leq m \leq M}$ is $\Omega f_{\mathrm{b}}^{\text {ext }}$ with

$$
\begin{aligned}
a_{m}= & \sqrt{\frac{2}{\kappa_{m}}}\left[2 \operatorname { s i n } ( C _ { \mathrm { h } } \frac { \kappa _ { m } } { 2 } ) \left(\cos \left(\kappa_{m}\left(L_{\mathrm{b}}-\mathbf{z}_{\mathrm{h}}\right)\right)+\cos \left(\kappa_{m} \mathbf{z}_{\mathrm{h}}\right) \cosh \left(\kappa_{m} L_{\mathrm{b}}\right)\right.\right. \\
& \left.-\sin \left(\kappa_{m} \mathbf{z}_{\mathrm{h}}\right) \sinh \left(\kappa_{m} L_{\mathrm{b}}\right)\right)-2 \sinh \left(C_{\mathrm{h}} \frac{\kappa_{m}}{2}\right)\left(\cosh \left(\kappa_{m}\left(L_{\mathrm{b}}-\mathbf{z}_{\mathrm{h}}\right)\right)\right. \\
& \left.\left.+\cos \left(\kappa_{m} L_{\mathrm{b}}\right) \cosh \left(\kappa_{m} \mathbf{z}_{\mathrm{h}}\right)+\sin \left(\kappa_{m} L_{\mathrm{b}}\right) \sinh \left(\kappa_{m} \mathbf{z}_{\mathrm{h}}\right)\right)\right] \\
b_{m}= & \kappa_{m} L_{\mathrm{b}}\left(\cos \left(2 \kappa_{m} L_{\mathrm{b}}\right)-2+\cosh \left(2 \kappa_{m} L_{\mathrm{b}}\right)\right) \\
& -\cosh \left(\kappa_{m} L_{\mathrm{b}}\right)\left(2 \sin \left(\kappa_{m} L_{\mathrm{b}}\right)+\cosh \left(\kappa_{m} L_{\mathrm{b}}\right) \sin \left(2 \kappa_{m} L_{\mathrm{b}}\right)\right) \\
{[\Omega]_{m}=} & \frac{a_{m}}{\sqrt{b_{m}}}
\end{aligned}
$$

\section{References}

[1] J. Chabassier, A. Chaigne, P. Joly, Modeling and simulation of a grand piano, The Journal of the Acoustical Society of America 134 (1) (2013) 648-665.

[2] C. Desvages, S. Bilbao, Physical modeling of nonlinear player-string interactions in bowed string sound synthesis using finite difference methods, in: Proceedings of the International Symposium on Musical Acoustics, 2014.

[3] S. Bilbao, A. Torin, Numerical simulation of string/barrier collisions: The fretboard, in: Proceedings of the 17th International Conference on Digital Audio Effects (DAFx-14), 2014.

[4] S. Bilbao, A. Torin, V. Chatziioannou, Numerical modeling of collisions in musical instruments, Acta Acustica united with Acustica 101 (1) (2015) $155-173$.

[5] V. Välimäki, J. Pakarinen, C. Erkut, M. Karjalainen, Discrete-time modelling of musical instruments, Reports on progress in physics 69 .

[6] S. Petrausch, Block based physical modeling, Ph.D. thesis, Ph. D. dissertation, University of Erlangen Nuremberg (2007).

[7] A. Fettweis, Wave digital filters: Theory and practice, Proceedings of the IEEE 74 (2) (1986) 270-327. 
[8] J. O. Smith, Physical modeling using digital waveguides, Computer music journal (1992) 74-91.

[9] A. Fettweis, Pseudo-passivity, sensitivity, and stability of wave digital filters, Circuit Theory, IEEE Transactions on 19 (6) (1972) 668-673.

[10] B. Maschke, A. J. Van Der Schaft, P. C. Breedveld, An intrinsic hamiltonian formulation of network dynamics: Non-standard poisson structures and gyrators, Journal of the Franklin institute 329 (5) (1992) 923-966.

[11] A. van der Schaft, Port-Hamiltonian systems: an introductory survey, in: Proceedings of the International Congress of Mathematicians, Madrid, $\mathrm{Au}-$ gust 22-30, 2006, 2007, pp. 1339-1365.

[12] V. Duindam, A. Macchelli, S. Stramigioli, H. Bruyninckx, Modeling and Control of Complex Physical Systems: The Port-Hamiltonian Approach, Springer Science \& Business Media, 2009.

[13] J. E. Marsden, T. Ratiu, Introduction to mechanics and symmetry: a basic exposition of classical mechanical systems, Vol. 17, Springer Science \& Business Media, 2013.

[14] S. Aoues, Schémas d'intégration dédiés à l'étude, l'analyse et la synthèse dans le formalisme hamiltonien à ports, Ph.D. thesis, INSA de Lyon (2014).

[15] N. Lopes, T. Hélie, A. Falaize, Explicit second-order accurate method for the passive guaranteed simulation of port-Hamiltonian systems, in: 5th IFAC Workshop on Lagrangian and Hamiltonian Methods for Non Linear Control (Lyon, France), 2015.

[16] A. Falaize, T. Hélie, Guaranteed-passive simulation of an electromechanical piano: A port-hamiltonian approach, in: 18th Int. Conference on Digital Audio Effects (DAFx-15), 2015.

[17] R. K. Instruments, Service Manual, CBS Musical Instruments, a division of CBS Inc., California, USA, 1979. URL http: //www . fenderrhodes . com/

[18] H. C. Hart, M. W. Fuller, W. S. Lusby, A precision study of piano touch and tone, The Journal of the Acoustical Society of America 6 (2) (1934) 80-94.

[19] M. Muenster, F. Pfeifle, T. Weinrich, M. Keil, Nonlinearities and selforganization in the sound production of the rhodes piano, The Journal of the Acoustical Society of America 136 (4) (2014) 2164-2164.

[20] M. Muenster, F. Pfeifle, Non-linear behaviour in sound production of the rhodes piano, in: Proceedings of the International Symosium of Musical Acoustics (ISMA). 
[21] A. van der Schaft, D. Jeltsema, Port-Hamiltonian Systems Theory: An Introductory Overview, Now Publishers Inc, 2014.

[22] H. K. Khalil, J. Grizzle, Nonlinear systems, Vol. 3, Prentice hall New Jersey, 1996.

[23] A. Stulov, Experimental and theoretical studies of piano hammer, in: Proceedings of the Stockholm Music Acoustics Conference, 2003.

[24] K. F. Graff, Wave motion in elastic solids, Dover publication, New York, 1973.

[25] S. G. Kelly, Advanced vibration analysis, CRC Press, 2006.

[26] K. T. McDonald, Electric guitar pickups, in: Pedagogic note, Princeton

490 University, Department of Physics, 2007, http://puhep1.princeton.edu/ mcdonald/examples/guitar.pdf.

[27] N. H. Fletcher, T. D. Rossing, The physics of musical instruments, Springer Science \& Business Media, 1998.

[28] N. Giordano, J. Winans II, Piano hammers and their force compression characteristics: Does a power law make sense?, The Journal of the Acoustical Society of America 107 (4) (2000) 2248-2255.

[29] M. Ghosh, Experimental study of the duration of contat of an elastic hammer striking a damped pianoforte string.

[30] X. Boutillon, Model for piano hammers: Experimental determination and digital simulation, The Journal of the Acoustical Society of America 83 (2) (1988) $746-754$.

[31] A. Stulov, Hysteretic model of the grand piano hammer felt, The Journal of the Acoustical Society of America 97 (4) (1995) 2577-2585.

[32] J. Chabassier, Modélisation et simulation numérique d'un piano par modèles physiques, Ph.D. thesis, Ecole Polytechnique X (2012).

[33] A. Macchelli, C. Melchiorri, Modeling and control of the timoshenko beam. the distributed port hamiltonian approach, SIAM Journal on Control and Optimization 43 (2) (2004) 743-767.

[34] J. A. Villegas, A port-Hamiltonian approach to distributed parameter systems, Ph.D. thesis (2007).

[35] V. Chatziioannou, M. Van Walstijn, An energy conserving finite difference scheme for simulation of collisions, in: Proceedings of the sound and music computing conference (SMC2013), Stockholm, 2013, pp. 584-591.

[36] R. F. Curtain, H. Zwart, An introduction to infinite-dimensional linear systems theory, Vol. 21, Springer Science \& Business Media, 2012. 
[37] T. Hélie, D. Matignon, Nonlinear damping models for linear conservative mechanical systems with preserved eigenspaces: a port-hamiltonian formulation, IFAC-PapersOnLine 48 (13) (2015) 200-205.

[38] L. Meirovitch, Principles and techniques of vibrations, Vol. 1, Prentice Hall New Jersey, 1997.

[39] N. G. Horton, T. R. Moore, Modeling the magnetic pickup of an electric guitar, American journal of physics 77 (2) (2009) 144-150.

[40] R. C. Paiva, J. Pakarinen, V. Välimäki, Acoustics and modeling of pickups, Journal of the Audio Engineering Society 60 (10) (2012) 768-782.

[41] L. Remaggi, L. Gabrielli, R. C. D. de Paiva, V. Välimäki, S. Squartini, A pickup model for the clavinet, in: Digital Audio Effects Conference (DAFx12), 2012.

[42] D. C. Hamill, Lumped equivalent circuits of magnetic components: the gyrator-capacitor approach, IEEE transactions on power electronics 8 (2) (1993) 97-103.

[43] D. C. Hamill, Gyrator-capacitor modeling: a better way of understanding magnetic components, in: Proceedings of the Applied Power Electronics Conference and Exposition (APEC'94), Ninth Annual, IEEE, 1994, pp. $326-332$.

535 [44] J. Cervera, A. Van Der Schaft, A. Baños, Interconnection of portHamiltonian systems and composition of dirac structures, Automatica 43 (2) (2007) 212-225.

[45] T. Itoh, K. Abe, Hamiltonian-conserving discrete canonical equations based on variational difference quotients, Journal of Computational Physics 76 (1) (1988) 85-102.

[46] G. Quispel, G. S. Turner, Discrete gradient methods for solving odes numerically while preserving a first integral, Journal of Physics A: Mathematical and General 29 (13) (1996) L341.

[47] R. I. McLachlan, G. Quispel, N. Robidoux, Geometric integration using discrete gradients, Philosophical Transactions of the Royal Society of London A: Mathematical, Physical and Engineering Sciences 357 (1754) (1999) 1021-1045.

[48] C. J. Budd, M. D. Piggott, Geometric integration and its applications, Handbook of numerical analysis 11 (2003) 35-139.

[49] J. D. Lambert, Computational methods in ordinary differential equations, Wiley London, 1973.

[50] A. Thorin, X. Boutillon, X. Merlhiot, J. Lozada, A non-smooth simulation of the dynamics of the grand piano action, in: Waves 2013, 2013. 